ARTICLE

\title{
Hepatitis $C$ virus has a genetically determined lymphotropism through co-receptor B7.2
}

Chia-Lin Chen ${ }^{1}$, Jeffrey Y. Huang ${ }^{1}$, Chun-Hsiang Wang ${ }^{1}$, Stanley M. Tahara ${ }^{1}$, Lin Zhou ${ }^{1}$, Yasuteru Kondo ${ }^{1}$ Joel Schechter ${ }^{2}$, Lishan Sư ${ }^{3}$, Michael M.C. Lai ${ }^{1,4}$, Takaji Wakita ${ }^{5}$, François-Loïc Cosset $^{6}$, Jae U. Jung ${ }^{1}$ \& Keigo Machida ${ }^{1}$

B-cell infection by hepatitis C virus ( $\mathrm{HCV}$ ) has been a controversial topic. To examine whether $\mathrm{HCV}$ has a genetically determined lymphotropism through a co-receptor specific for the infection by lymphotropic HCV, we established an infectious clone and chimeric virus of hepatotropic and lymphotropic HCV strains derived from an HCV-positive B-cell lymphoma. The viral envelope and 5'-UTR sequences of the lymphotropic HCV strain were responsible for the lymphotropism. Silencing of the virus sensor, RIGI, or overexpression of microRNA-122 promoted persistent viral replication in B cells. By cDNA library screening, we identified an immune cell-specific, co-stimulatory receptor B7.2 (CD86) as a co-receptor of lymphotropic $\mathrm{HCV}$. Infection of B cells by HCV inhibited the recall reaction to antigen stimulation. Together, a co-receptor B7.2 enabled lymphotropic HCV to infect memory B cells, leading to inhibition of memory B-cell function and persistent HCV infection in HCV-infected hosts.

\footnotetext{
${ }^{1}$ Department of Molecular Microbiology and Immunology, Keck School of Medicine, University of Southern California, 2011 Zonal Avenue, Los Angeles, California 90033, USA. ${ }^{2}$ Department of Cell and Neurobiology, Keck School of Medicine, University of Southern California, 2011 Zonal Avenue, Los Angeles, California 90033, USA. ${ }^{3}$ Department of Microbiology and Immunology, Lineberger Comprehensive Cancer Center, University of North Carolina at Chapel Hill, Chapel Hill, North Carolina 27599-7290, USA. ${ }^{4}$ Institute of Molecular Biology, Academia Sinica, Taipei 115, Taiwan. ${ }^{5}$ Department of Virology II, National Institute of Infectious Diseases, Tokyo 162-8640, Japan. ${ }^{6}$ International Center for Infectiology Research, Team EVIR, Inserm, U1111, Université Claude Bernard Lyon 1, CNRS, UMR5308, Ecole Normale Supérieure de Lyon, Univ Lyon, F-69007 Lyon, France. Correspondence and requests for materials should be addressed to K.M. (email: keigo.machida@med.usc.edu).
} 
epatitis $\mathrm{C}$ virus (HCV) infection often persists despite robust host immune responses, consequently leading to chronic hepatitis, liver cirrhosis and hepatocellular carcinoma. However, $\mathrm{HCV}$ infection and replication in immune cells remains controversial and is not universally accepted. Even though experimental and clinical evidence accumulated during the last two decades are compelling, the issue remains controversial mainly due to insufficient information and deeply fragmented knowledge. Another potentially serious complication of $\mathrm{HCV}$ infection is the possible infection of peripheral blood mononuclear cells (PBMC) by HCV leading to B-lymphocyte proliferative disorders, including mixed cryoglobulinemia, oligoclonal proliferation of $\mathrm{B}$ cells ${ }^{1,2}$, and B-cell non-Hodgkin's lymphoma ${ }^{2-5}$. Still, HCV infection of B cells and its possible association with B-cell disorders remains a controversial subject $^{6,7}$.

It was reported from McKeating's group that HCV replication in lymphocytes is relatively rare and attachment of HCV particles to $\mathrm{B}$ lymphocytes did not lead to productive $\mathrm{HCV}$ replication ${ }^{7}$. HCV promoted the adhesion of primary B cells to Huh-7 cells for retention of $B$ cells on infected hepatocytes, thus implying that $\mathrm{B}$ cells may provide a vehicle for HCV persistence by transmission to the liver. Additionally, lymphotropism of HCV (SB strain: patient splenoma B-cell-derived isolated by our group) is not limited to B cells since we have identified HCV infection (SB strain) of $\mathrm{T}$ cells and subsequent alterations in their functions ${ }^{8,9}$. These studies, however, did not provide conclusive evidence that other molecules on other immune cell types serve as HCV co-receptors.

Cellular surface receptors have been identified as factors promoting viral tropism. HCV uses cell surface factors (LDL-R and HSPG) (ref. 10) for attachment and additional entry factors for infection of hepatocytes. The entry factors include the Scavenger Receptor class B type I (SRB1 or SR-BI) (ref. 11), the tetraspanin CD81 (ref. 12), the tight junction proteins CLDN1 (ref. 13) and the receptor tyrosine kinases EGFR and EphA2 (ref. 14). More recently, the Niemann-Pick C1-like 1 (NPC1L1) cholesterol absorption receptor and the iron uptake receptor transferrin receptor 1 (TfR1) have also been shown to play a role in HCV entry ${ }^{15}$. Among these, four co-receptors (Claudin-1, Occludin, CD81 and SR-BI) are potentially involved in HCV entry $^{12,16-18}$, while sulfated homologues of heparin inhibit HCV entry into mammalian cells ${ }^{19}$. These co-receptors are associated with the viral envelope glycoprotein of HCV. The viral envelope proteins include E1 and E2, which assemble as heterodimers in the prebudding virion form ${ }^{20}$.

Mutations in the $5^{\prime}$-UTR ( $5^{\prime}$-untranslated region) of a hepatotropic HCV strain (H77) cultured in T lymphoid cell lines enhanced viral replication specifically in $\mathrm{T}$ lymphoid cells (MOLT-4) (ref. 21). The presence of different, strain-specific $5^{\prime}$-UTR sequences or sequence heterogeneities in the region coding for E1 or E2 can result in altered lymphotropism when compared to hepatotropic strains ${ }^{22,23}$. However, the lymphotropism of these viruses and the significance of these sequence variations were not fully established since only three nucleotide substitutions found in the $5^{\prime}$-UTR in hepatotropic JFH1 strain and variant H77 strain passaged in lymphocytes are unchanged. The sequence variations in the $5^{\prime}$-UTR region are usually associated with $\mathrm{B}$ and $\mathrm{T}$ lymphocyte replication of $\mathrm{HCV}$ (refs 21,24,25). It has been shown that the B-cell specific $5^{\prime}$-UTR has a lower translation difference observed between lymphotropic and hepatotropic strains ${ }^{26}$. (The lymphotropic strain may have a less efficient $5^{\prime}$-UTR for translation).

The viral envelope protein is frequently mutated in chronically infected subjects, whereas the $5^{\prime}$-UTR of HCV RNA in B cells is not frequently mutated $^{22}$, suggesting that $\mathrm{B}$ cells suppress replication of less competent viral sequences whereas liver cells are not as stringent. In SCID mice inoculated with experimentally mutated HCV strains, the HCV mutations in the $5^{\prime}$-UTR (specifically the IRES) or C-E1-E2 regions have different consequences on genome replication and/or translational activity as a function of cell type ${ }^{26,27}$. Moreover, the $3^{\prime}$-UTR also contributes to efficient RNA replication of HCV (ref. 28). The $3^{\prime}$-end of the NS5B-coding region has been shown to form part of a secondary structure (kissing loop) involving the $3^{\prime}$-UTR (ref. 28). This fact may explain why the HCV pseudoparticle (HCVpp) bearing HCV envelope alone did not confer robust replication ability in $\mathrm{B}$ cells.

MicroRNA-122 (miR-122) is the most abundant miRNA species in liver and binds the $5^{\prime}$-UTR of HCV genomic RNA to facilitate HCV replication. Chronic liver damage results in release of miR-122 into the blood stream and exposes miR-122 to immune cells. However, the role of miR-122 for HCV replication in immune cells is not documented. MicroRNA-122 is not only expressed in the liver but also in malignant and chemotherapytreated lymphocytes ${ }^{29,30}$, and positively regulates HCV replication in hepatocytes ${ }^{31-33}$. Circulating miR-122 can be taken up by several cell types, including lymphocytes ${ }^{34,35}$ thereby facilitating HCV replication. We hypothesized that this uptake of miR-122 facilitates HCV replication in B cells. Indeed, circulating plasma miR-122 in exosomes is elevated in early stage fibrosis induced by various aetiologies, including HCV infection, hepatocyte injury and inflammation ${ }^{36-39}$.

In this study, we hypothesized that the ability of certain HCV strains to infect B cells was determined by the sequence variation of the viral genome. We used genetic approaches to show that different $\mathrm{HCV}$ strains vary in their relative lymphotropism or hepatotropism. The B-cell infection by lymphotropic HCV may play a role in $\mathrm{HCV}$ pathogenesis. These results are crucial for studying the natural history of HCV infections in order to gauge the full scope of its subsequent pathological consequences resulting from $\mathrm{HCV}$ infections. We investigated the roles of plasma miR-122 in HCV pathogenesis, especially in lymphocytes $^{31}$ and the role of exosome-mediated delivery of tropic factors in creating a permissive environment for viral infection and replication.

\section{Results}

Lymphotropic SB-HCV strain encodes a unique E1-E2. We have previously isolated an $\mathrm{HCV}$ clone (SB-HCV) from an $\mathrm{HCV}$-infected $(+)$ B-cell lymphoma line $\mathrm{e}^{40}$. This virus variant can infect $B$ cells in vitro ${ }^{40}$; however, virus production was low for reasons unclear. To answer this question we used a genetic approach and assembled the full-length SB viral genomic RNA (genotype $2 b$ ) cloned from HCV infected SB cells ${ }^{40}$. Viral RNA was cloned in 14 overlapping cDNA fragments using primer sets based on the consensus sequences (Supplementary Fig. 1). Sequence determination of the resulting full-length, HCV cDNA showed that the SB-HCV strain represented a unique HCV isolate.

To determine whether unrelated HCV strains replicate to differing extents in B cells, we compared the full-length RNAs from the SB and JFH-1 strains, the latter of which is a wellestablished hepatotropic strain ${ }^{41}$, by in vitro transfection of either Huh7.5.1 cells (a hepatocellular carcinoma cell line with inactivating mutations in RIG-I) or Raji cells (a human B-lymphoid cell line) (Fig. 1). In Raji cells, the SB viral RNA titer remained at $10^{4}-10^{5}$ copies $\mu g^{-1}$ of RNA at day 20 posttransfection. For the corresponding RNA polymerase active site (GND) mutant RNA, which contains an inactivating mutation of NS5B (replication incompetent virus) (Fig. 1a), viral RNA 
a

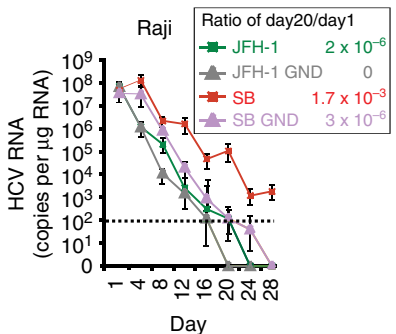

Day b

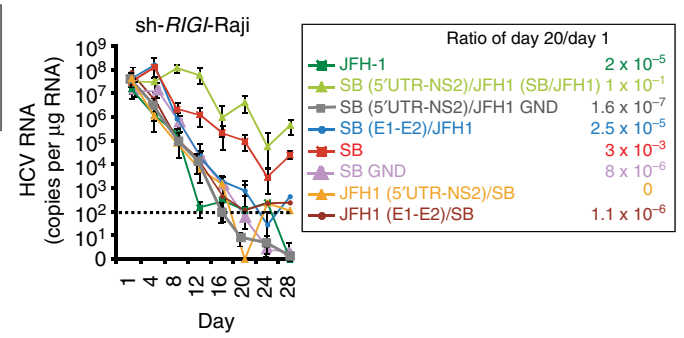

C

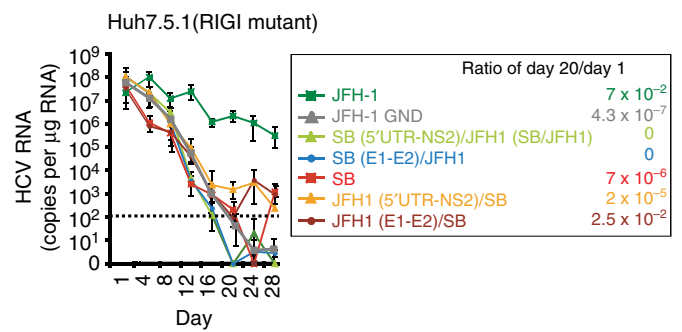

d

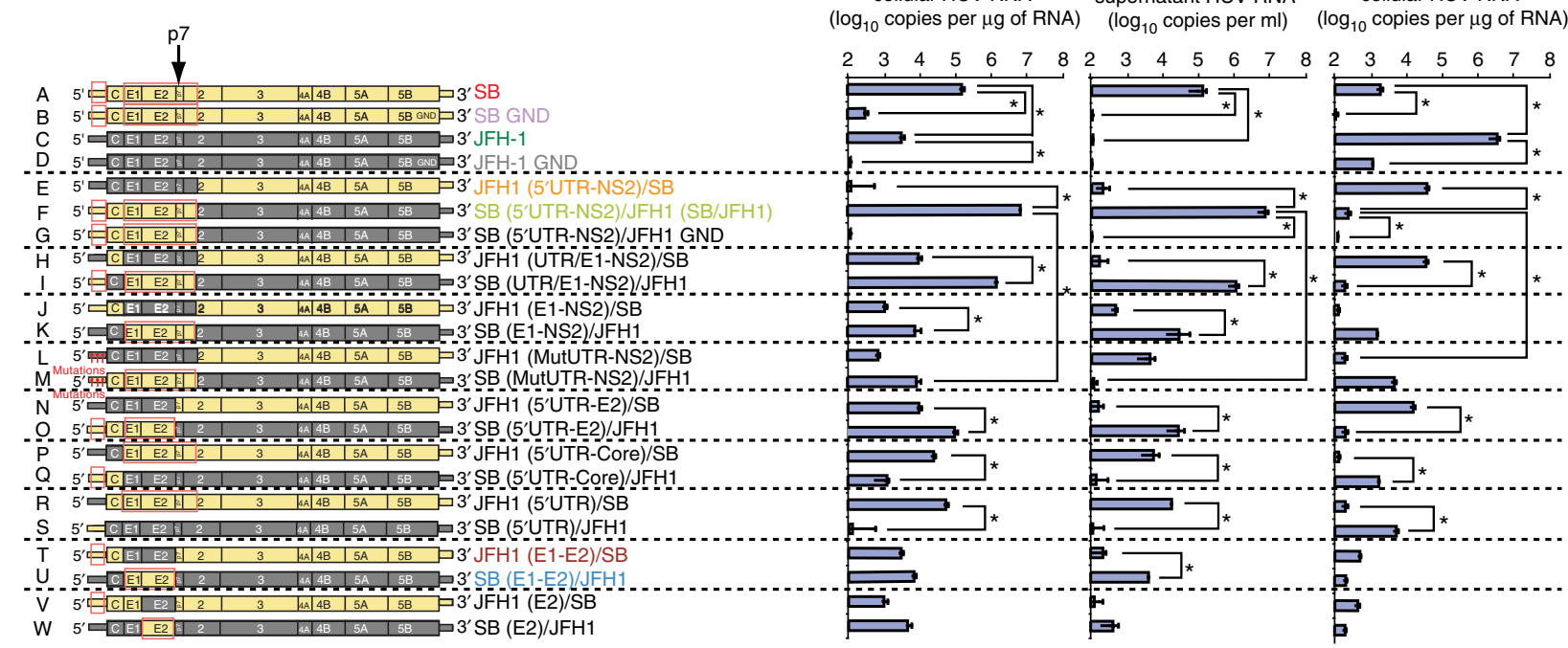

Figure 1 | Genetic determinants of tissue tropism of HCV SB and JFH1 viruses. (a-c) HCV virus RNA in the parental Raji cells (a), RIG-I knockdown Raji (sh-RIGI-Raji) cells (b) or Huh7.5.1 cells (c). Cells were harvested from day 1 until day 28 after HCV RNA transfection. The HCV RNA titers were determined by qRT-PCR (performed in quadruplicates, $n=3$ ). Error bars represent s.d. (d) Genetic structure of virus chimera assembled from the SB and JFH-1 strains. The chimeric viruses were transfected into Raji (sh-RIGI-Raji) or Huh7.5.1 cells as indicated; cellular HCV RNA was quantitated by qRT-PCR at 24 days after transfection (Left and Right). The HCV RNA titers in the supernatant are shown in (d, middle). HCV SB, but not commonly studied JFH-1 strain, preferentially infects, replicates and produces virion particles in sh-RIGI-Raji lymphoma B cells, but not in Huh7.5 hepatocyte cells ( $n=3$ ). Error bars represent s.d.

decreased over three weeks of incubation. By comparison, the RNA titer of the JFH-1 strain quickly declined in Raji cells. These results indicated that the SB-HCV strain, but not the JFH-1 strain, was able to replicate or was unstable RNA in Raji cells. Conversely, in Huh7.5.1 cells (Fig. 1c), the SB-HCV strain was less efficiently replicated by day 28 post-transfection when compared to Raji cells (Fig. 1a); this indicated that Raji cells are more permissive for SB strain replication than Huh7.5.1. cells.

During the chronic HCV infection stage, the RIGI RNA sensor pathway is impaired because HCV protease NS3/4A cleaves the RIGI adaptor protein MAVS (IPS-1) (refs 42,43). Furthermore, chronic inflammatory cytokines induce negative feedback pathways through induction of NLRX1 (refs 44,45) to moderate the excessive activation of RIGI pathways. To study how HCV interacts with B cells, Raji cells were infected with a lentiviral vector expressing shRNA targeting RIGI to reduce the antiviral defences (Fig. 1b). Indeed, silencing RIGI in Raji cells significantly increased HCV RNA levels and reduced interferon (IFN)- $\beta$ induction probably due to defective viral RNA recognition and induction of RIGI downstream genes (that is, IFN- $\beta$ ). In contrast, silencing of MDA5, another innate immune receptor, did not have any further effect (Supplementary Fig. 2), which was consistent with the finding that HCV RNA binds to RIGI, but not to MDA5 (ref. 46). The highly permissive hepatotropic liver cell line (Huh7.5.1), which is highly permissive for hepatotropic
HCV, has an amino acid replacement (T55I) in the first CARDhomology domain ${ }^{47}$ of RIGI, resulting in inactivation of RIGI function. Therefore, the tropism of HCV-SB and hepatotropic strains was compared under identical RIGI-deficient conditions (Fig. 1b,c).

To identify the viral gene or genome region(s) responsible for the differential cellular tropism of these viral strains, we constructed chimeric virus genomes from the lymphotropic SB-HCV and hepatotropic JFH-1 HCV strains (Fig. 1d). These constructs were then converted into full-length infectious RNA for transfection into recipient cells (Fig. 1d). From the results of the various viral genome chimeras, it was apparent that all chimeric genomes containing the SB $5^{\prime}$-UTR and homologous structural proteins replicated well in sh-RIGI-Raji cells and released viral RNA (some of them are associated with virion) into the cell culture supernatant. In particular, a chimera containing the JFH1 backbone and the entire SB 5'-UTR-Core-E1-E2p7-NS2 (construct F: at the second transmembrane domain of NS2) replicated 50-fold more robustly than the parental SB virus in sh-RIGI-Raji cells, producing nearly $10^{7} \mathrm{HCV}$ RNA copies $\mathrm{ml}^{-1}$. By contrast replication-incompetent SB/JFH1 GND mutant virus RNA levels were significantly lower (construct G). The corresponding reciprocal genome constructs containing E1-E2 and the $5^{\prime}$-UTR from JFH1 (construct E) replicated poorly in sh-RIGI-Raji cells. In Huh7.5.1 cells, 
this result was reversed: JFH-1 RNA replicated to a level of $10^{7}$ RNA copies $\mathrm{ml}^{-1}$, whereas SB RNA replication was decreased to a significantly lower level $(P<0.05, t$-test $)$. Furthermore, mutations in the $5^{\prime}$-UTR regions of SB and JFH1 strains (constructs $\mathrm{L}$ and $\mathrm{M}$ ) resulted in significantly reduced HCV RNA levels in lymphocytes. These results suggested that there are distinct hepatotropic and lymphotropic HCV strains, whose tropism is largely determined by the envelope proteins (E1-E2) and their respective $5^{\prime}$-UTRs. This dramatic increase was probably due to the sequence changes that occurred at a crucial junction between the second transmembrane segments of NS2 as previously suggested ${ }^{48}$.

To further ascertain the importance of E1-E2 proteins in the lymphotropism of SB virus, we produced HCVpp using an envelope-defective HIV-1 proviral vector ${ }^{49-51}$. This HCVpp bears the E1 and E2 envelope proteins of HCV and contains a luciferase reporter gene in the lentivector. As shown in Fig. 2a, HCVpp bearing the SB E1-E2 envelope proteins could efficiently infect Raji cells, whereas its infectivity of Huh7.5.1 cells was significantly lower. As a control, HCVpp assembled with either
E1 or E2 proteins alone were not infectious. The infectivity of HCVpp with SB envelope protein in Raji cells was substantially lower (more than 3-logs lower) than that of HIV vector pseudotyped with MLV Env. Addition of neutralizing anti-E2 antibody (CBH5 clone) specifically antagonized HCVpp entry into Raji cells (Fig. 2a, right). The ability of HCVpp-SB to infect Raji cells gives further support that the SB strain is lymphotropic while HCVpp-JFH1 do not infect Raji cells. Indeed, in the CD81-interacting domain of the E2 region ${ }^{52-54}$, the SB-HCV amino acid sequence is homologous to other HCV strains, but does have several unique residues that may be responsible for the observed lymphotropism (Fig. 2c). Another genotype $2 \mathrm{~b}$ strain ( $\mathrm{AB}$ 30907) has the highest homology to the $\mathrm{SB}$ strain among all HCV genotypes while the H77 genotype 1 strain has lesser homology with the SB strain (Fig. 2b,c). Amino acids G496 and N514 of SB HCV E2 are unique amino acids in comparison to other HCV E2 sequences, which contain S514 (ref. 55). The G496C substitution has been shown to form a disulfide bond with C567 (ref. 55). Substitution of G496 by Cys significantly reduced SB E2 binding to B cells while this a

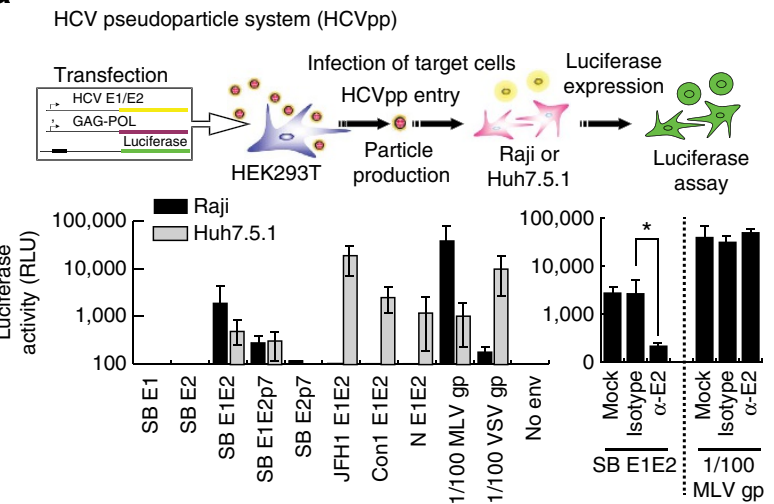

b
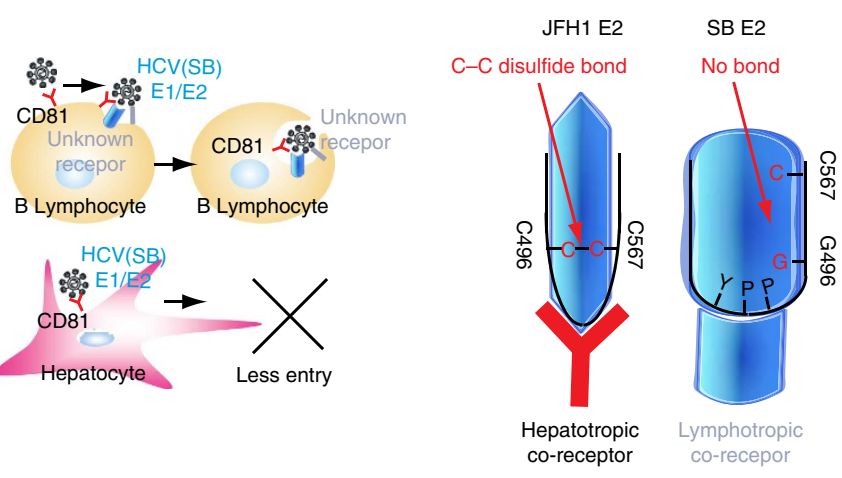

C
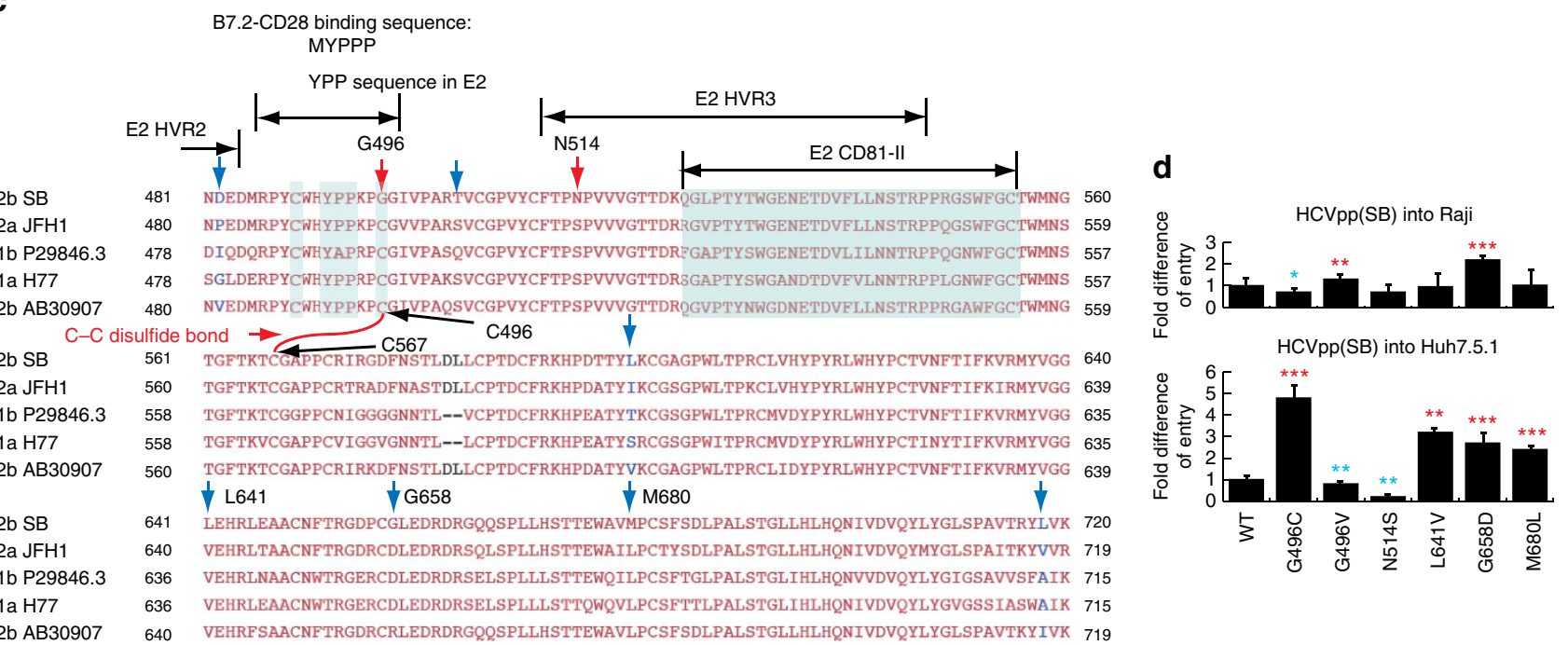

Figure 2 | Lymphotropic pseudoparticles (HCVpp) can infect B lymphocytes more efficiently than hepatic cells. (a) HIV-luc vector was pseudotyped with envelope proteins of HCV SB strain. Pseudoparticles (HCVpp) efficiently infected B lymphocytes, but not hepatic cells. Infectivity of HCV pseudo-particles was measured by luciferase assay. HIV vector containing a luciferase reporter pseudotyped with E1 and/or E2 of SB strain, Con1, or HCV-N strain and MLV glycoprotein (MLV gp), or no envelope (no env) was used to infect Raji (black bars) or Huh7.5.1 (grey bars). Luciferase activity was assayed at 4 days after infection. Addition of neutralizing anti-E2 antibody blocked HCVpp entry (a, right). Experiments were repeated at least three times ( ${ }^{\star} P<0.05$, T-test, $n=3$ ). Error bars represent s.d. (b) Hypothetical model of E2 sequence mutations on HCV B cell tropism. (c) The HCV E2 amino acids sequences are variable. Red arrows indicate the different amino acid positions between SB and other HCV strains. 1b strain is from sequence accession number P29846.3. (d) Variations of E2 sequences change HCV tropism ( ${ }^{\star} P<0.05$; ${ }^{\star \star} P<0.01$; ${ }^{\star \star} P P<0.001$, ANOVA, $n=3$ ). Error bars represent s.d. 
substitution significantly increased virus entry into Huh7 cells (Fig. 2d). Formation of the disulfide bonding between G496C and C567 may restore or stabilize a conformation favourable of E2 for entry into hepatocyte HCV co-receptors (Fig. 2d). These results confirmed that the E1-E2 envelope proteins conferred the observed lymphotropism exhibited by the SB strain in addition to the properties of strain-specific 5'-UTRs.

Envelope and $5^{\prime}$-UTR determine lymphotropism of SB virus. The predicted secondary structure of the SB $5^{\prime}$ UTR includes several possible RNA stem-loop structures (Fig. 3a). Five nucleotide differences between the SB and JFH1 strains are found within $5^{\prime}$-UTR and their positions are indicated (Fig. 3a). To address the significance of these genetic differences, and potential effects on RNA secondary structure, four out of five nucleotides were changed from the SB strain to those found in the JFH1 strain. Interestingly, the four-nucleotide substitutions significantly reduced HCV RNA levels in sh-RIGI-Raji cells at 20 days post transfection of HCV RNA (Fig. 3b). The A181G and U280C substitutions did not affect HCV RNA levels, but combined substitutions (A181G, U256G, C276A and U280C), and to a lesser extent, U256G and C276A substitutions reduced HCV RNA levels. However, it cannot be excluded that these differences may be due to changes in RNA stability. Therefore, we constructed a mutant subgenomic JFH1 replicon to see if substitutions of the $5^{\prime}$-UTR sequence promoted replication in B cells. The $5^{\prime}$-UTR sequences were replaced with SB $5^{\prime}$-UTR sequences of the JFH1 subgenomic HCV replicon. We observed that combined substitutions in G181A, G256U, A276C and C280U increased HCV RNA levels of the JFH1 subgenomic replicon (Fig. 3c) in sh-RIGI-Raji cells. These results indicated that nucleotides A181, U256, C276 and U280 of the $5^{\prime}$-UTR have key roles in regulating HCV levels in sh-RIGIRaji cells.

To prove that SB-HCV recombinants produced infectious virus particles, the culture supernatant from the transfected cells was used to infect naïve sh-RIGI-Raji or Huh7.5.1 cells by using a batch system to ensure a cell-free transfer (Supplementary Fig. 3). SB RNA-transfected Raji cells produced infectious virus that infected naïve sh-RIGI-Raji cells (Supplementary Fig. 3A and B), but was less efficient at replication in Huh7.5.1 cells (Supplementary Fig. 3C). The converse was true for the JFH strain in Huh7.5.1 cells (Supplementary Fig. 3C). Infectivity of $\mathrm{SB} / \mathrm{JFH} 1$ virus was directly quantified by limiting dilution assay, demonstrating that $\mathrm{HCV}$-infected cells have infectivity of $40 \%$ $(6 / 15$ clones) at day 13 and $38 \%$ (6/16 clones) at day 20 (Supplementary Fig. 3D). Infection of HCV did not significantly affect cell proliferation (Supplementary Fig. 3E).

The role of miR-122 in HCV lymphocyte infection was examined by transduction of miR-122 into B lymphocytes. A miR-122 expressing lentivirus was transduced into sh-RIGIRaji cells and cells were challenged with HCV (Fig. 3d). The results showed that miR-122 transduction significantly enhanced HCV replication, producing virus titers of $2 \times 10^{6}$ copies $\mathrm{ml}^{-1}$ and $10^{8}$ copies $\mu \mathrm{g}^{-1}$ of cellular RNA (387-fold increase: $P<0.001, t$-test) after 3 weeks post-transduction (Fig. 3e). Cellular miR-122 levels were significantly reduced 4 days post-transduction while levels of miR-122 significantly increased in the supernatant (Supplementary Fig. 4). Indeed, HCV RNA levels in culture supernatant significantly increased by day 7 and further increased at day 14 post-transfection $(P<0.05, t$-test, Supplementary Fig. 4 , right), indicating that miR122 may be secreted from HCV-infected B cells. Furthermore, miR-122 levels were measured in immune cells isolated from
HCV patients. Consistent with in vitro results, circulating miR-122 levels were significantly increased in HCV-infected patients $(P<0.05, t$-test, Fig. 3f $)$. The increased expression of miR-122 in B cells was confirmed in memory B cells from HCV patients $(n=9)$. The higher miR-122 levels in memory B cells showed a positive correlation with higher cellular HCV RNA levels ( $r=0.67, P<0.05, t$-test, Fig. $3 \mathrm{~g})$.

In order to determine if reduction of miR-122 levels had a negative effect on HCV replication in B cells, an antagomir of miR-122 was transfected into human memory B cells isolated from HCV patients and examined for effect on cellular levels of HCV RNA in B cells. These primary cells were incubated ex vivo to allow $\mathrm{HCV}$ replication. Interestingly, transfection of miR-122 antagomir into patients' memory B cells significantly lowered HCV replication $(P<0.05$, $t$-test; Fig. $3 \mathrm{~h})$.

Transfer of miR-122 via exosomes was tested in vitro for effect on HCV replication. Exosomes derived from HCV patients or healthy individuals were added to the supernatant of HCV-infected Raji cells and examined for effect on HCV replication. Addition of exosomes containing high miR-122 levels from HCV patients indeed enhanced HCV replication (14-fold increase of HCV RNA) in Raji cells (Fig. 3i). These results indicated that miR-122 promotes $\mathrm{HCV}$ replication in B lymphocytes.

CLDN1/OCLN can rescue hepatotropic HCV infection. We next studied the cellular basis for the lymphotropism of the SB virus by focusing on the role of CLDN1 and OCLN, which have been shown to be the co-receptors for HCV infection of Huh7.5.1 cells ${ }^{13,16}$. CLDN1 and OCLN are normally expressed at very low or undetectable levels in Raji cells (Fig. 4a) (ref. 56). To test whether overexpression of either CLDN1 or OCLN could render Raji cells more susceptible to infection by hepatotropic JFH-1, we overexpressed CLDN1 or OCLN in Raji cells. As shown in Fig. 4b, overexpression of CLDN1 or OCLN in Raji cells resulted in only a marginal increase of viral entry and not to the extent observed for SB (E1-E2). By comparison, HCVpp bearing E1-E2 of Con1 or JFH-1 could not infect Raji cells. It is not clear if lower infectivity was due to inability to adsorb and enter cells or due to lower efficiency of viral gene expression and replication. To test if HCV cell entry required both OCLN and CLDN1 co-receptors, both OCLN and CLDN1 were simultaneously transfected into Raji cells prior to challenge with HCVpp-JFH1. The extent of successful HCV intracellular entry was assessed by luciferase assay (Fig. 4c). As four human co-receptors (OCLN, CLDN1, CD81 and SR-BI) are critical for HCV entry into hepatocytes, the effect of OCLN and CLDN1 overexpression was examined in Raji cells, which normally lack their expression. Interestingly, Raji cells expressing both OCLN and CLDN1 were found to promote entry of hepatotropic JFH1 HCV (Fig. 4c), confirming that viral entry required co-expression of both OCLN and CLDN1. The intrinsic ability of HCVpp-SB to infect Raji cells, but not HCVpp-JFH1, in the absence of OCLN and CLDN1, to infect Raji cells gives further support to our contention that the SB strain is lymphotropic (while Huh7 cells express all four receptors CLDN1, OCLN, CD81 and SR-BI).

On the other hand, CLDN1, OCLN, SR-BI or CD81 knockdown in Huh7.5.1 cells potently inhibited the infection by HCVpp-JFH-1 (Fig. 4d,e), confirming the previous report describing essential roles of four co-receptors CLDN1, OCLN, CD81 and SR-BI for virus entry into hepatocytes ${ }^{12,13,16,57}$. In contrast, while the knockdown of CD81 or SR-BI inhibited HCVpp-SB infectivity in Raji cells, knockdown of CLDN1 or OCLN did not exhibit this inhibition. The results of Fig. 4d,e indicated that, in contrast to JFH1, SB strain entry is a CD81- and 
a

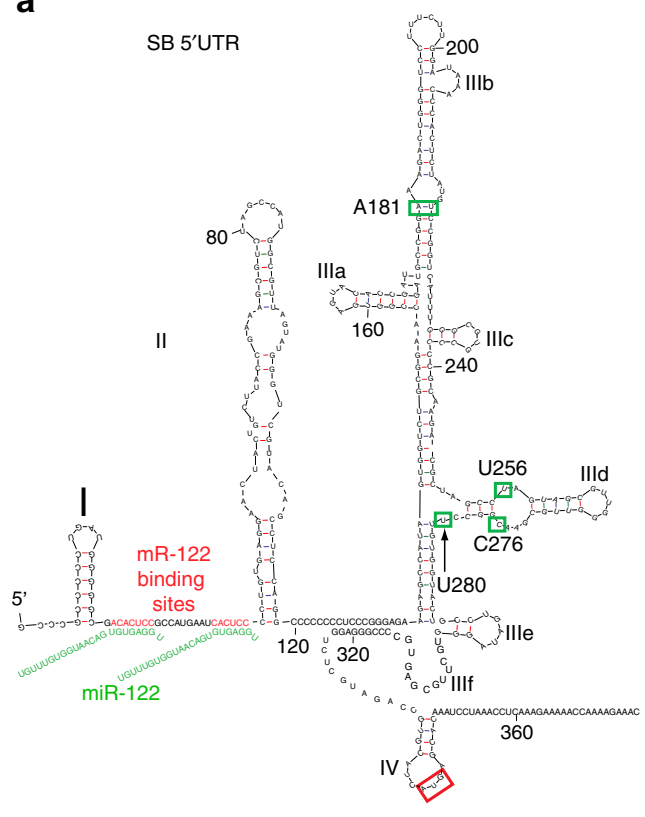

f

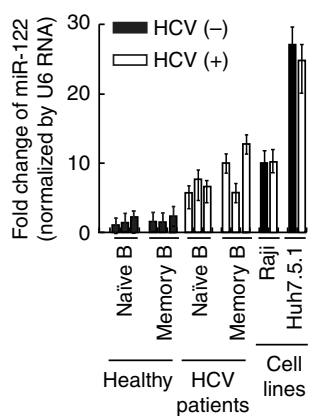

b

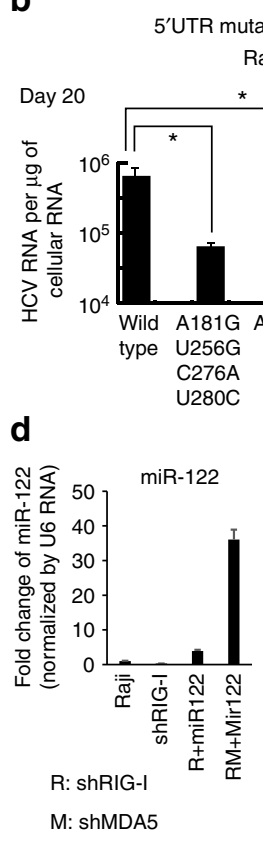

$280 \mathrm{C}$ c Raji-RIGI

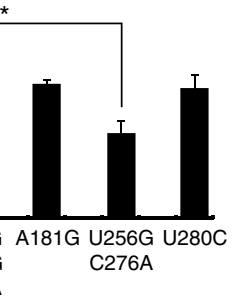

e

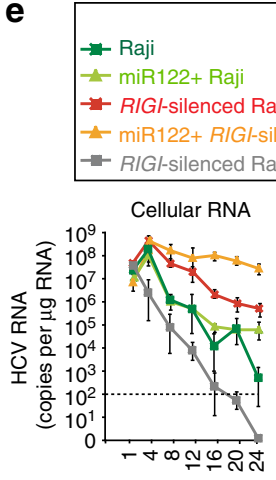

Day
5'UTR mutagenesis analysis in JFH1 subgenomic replicon

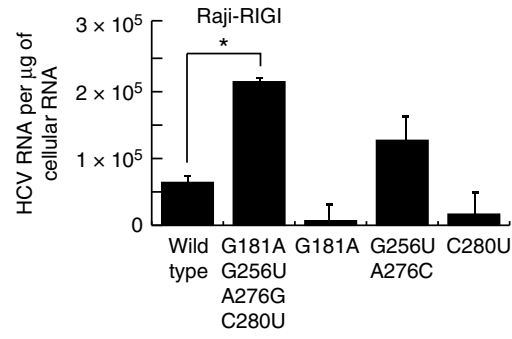

Ratio of day 20/day 1 cellular HCV RNA

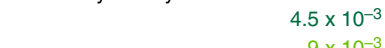

$9 \times 10^{-3}$

$2.3 \times 10^{-2}$

\section{enced Raji}

GND mutant

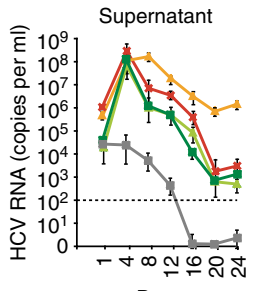

Day

h

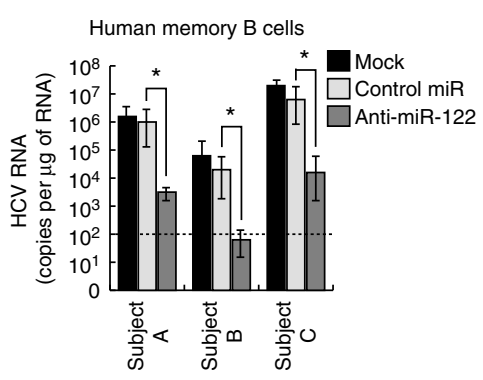

i

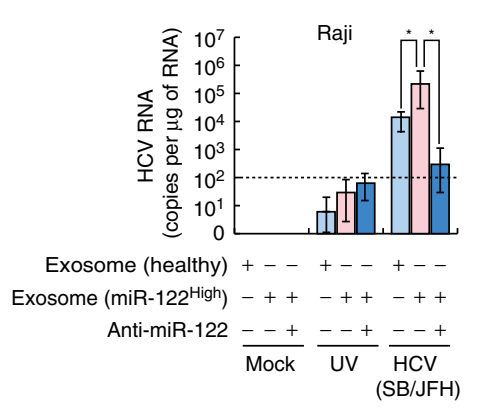

Figure 3 | Sequence of 5'-UTR of lymphotropic HCV and cellular miR-122 levels are involved in lymphotropism. (a) The putative structure of the $5^{\prime}$-UTR of SB-HCV RNA as determined by MFOLD program (computational secondary structure prediction software). The nucleotides in green boxes are those that differ between SB and JFH1 RNA. These nucleotides are located in stem-loops (IIla and IIId) and bulge regions, which may recruit lymphocyte-specific factors. The nucleotides in red boxes are the first AUG (start codon). miR-122 seed-match sequences (red) and predicted interactions with miR-122 (green) are shown. (b) The HCV RNA titers in sh-RIGI-Raji cells transfected with various $5^{\prime}$-UTR mutants of SB RNA ( ${ }^{\star} P<0.05$, ANOVA, $n=3$ ). Error bars represent s.d. (c) The HCV RNA titers in sh-RIGI-Raji cells transfected with the various $5^{\prime}$-UTR mutants of JFH1 subgenomic replicon RNA ( ${ }^{\star} P<0.05$, ANOVA, $n=3$ ). Error bars represent s.d. (d) miR-122 expression levels in Raji cells transduced with lentivirus expressing shRIGI, sh-MDA5 or mir-122 $(n=3)$. Error bars represent s.d. (e) Transduction of microRNA miR-122 enhances and stabilizes HCV replication in B lymphocytes. Effects of RIGI and miR-122 on lymphotropic HCV SB infection were examined using specific shRNA $(n=3)$. Error bars represent s.d. (f) Human memory B cells have higher levels of miR-122 in clinical specimens. Cellular miR-122 levels were determined by qRT-PCR in quadruplicate in PBMCs from three different patients $(n=3)$. Error bars represent s.d. (g) Analysis of HCV RNA in B cells versus miR-122 in B cells. The miR-122 levels of B cells from HCV patients were comparable to that of Raji cells. The miR-122 abundance is correlated with higher HCV RNA levels in memory B cells. Cellular HCV RNA levels were determined by qRT-PCR in nine different patient PBMCs. (h) Memory B cells from three patients were infected with HCV and cellular HCV RNA levels were determined by $q R T$-PCR in quadruplicate. ( ${ }^{\star} P<0.05$, Student $t$-test, $n=3$ ). Error bars represent s.d. (i) To determine if miR- 122 sequestration reduced HCV replication in B cells, antagomir of miR-122 was transfected into human memory B cells isolated from HCV patients and examined for cellular levels of HCV RNA in B cells ( ${ }^{\star} P<0.05, t$-test, $\left.n=3\right)$. Error bars represent s.d.

SR-BI-dependent but CLDN1- and OCLN-independent pathway for infection of B cells.

B7.2 is a co-receptor for lymphotropic $\mathrm{HCV}$ of B cells. Inability of SB to infect hepatocytes indicated that some other cell surface co-receptor proteins were needed. In an effort to identify the potential novel co-receptor specific for lymphotropic HCV infection, we performed a lentivirus-based screen of a cDNA library, derived from the highly HCV-permissive B lymphoma cell lines Raji and Daudi. This screen was designed for identification of genes that rendered the non-permissive $\mathrm{CD} 81^{+}$ $\mathrm{SR} \mathrm{BI}^{+}$HEK293T cell line susceptible to infection by HIV-luc reporter vector pseudotyped with HCVgp (HCVpp) (Fig. 5a). The result of this functional screen identified ten co-receptor candidate genes (Supplementary Fig. 5), which were tested in 
a

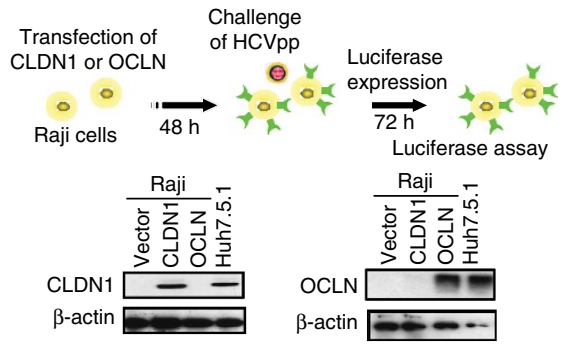

b

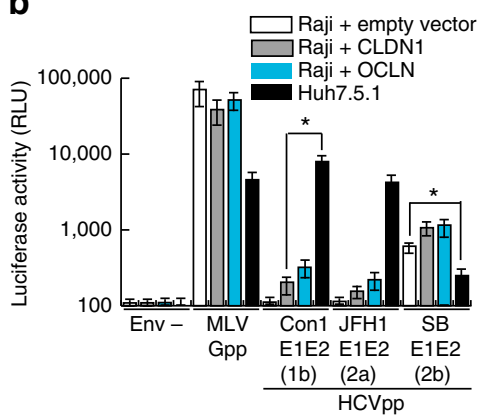

\section{C}

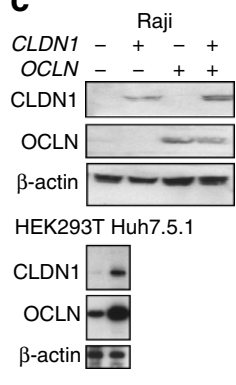

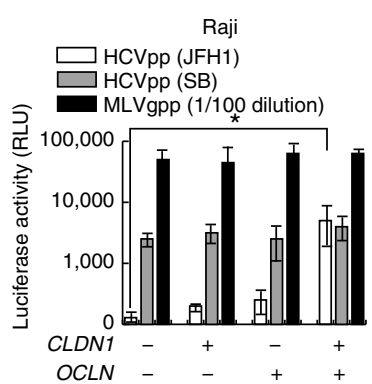

d

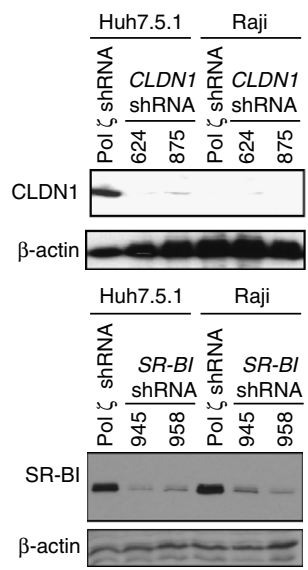

e

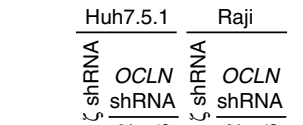

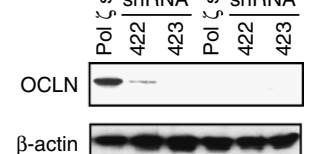

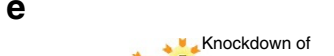

Challenge

Challenge
of HCVpp

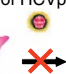

$72 \mathrm{~h}$ Luciferase assay
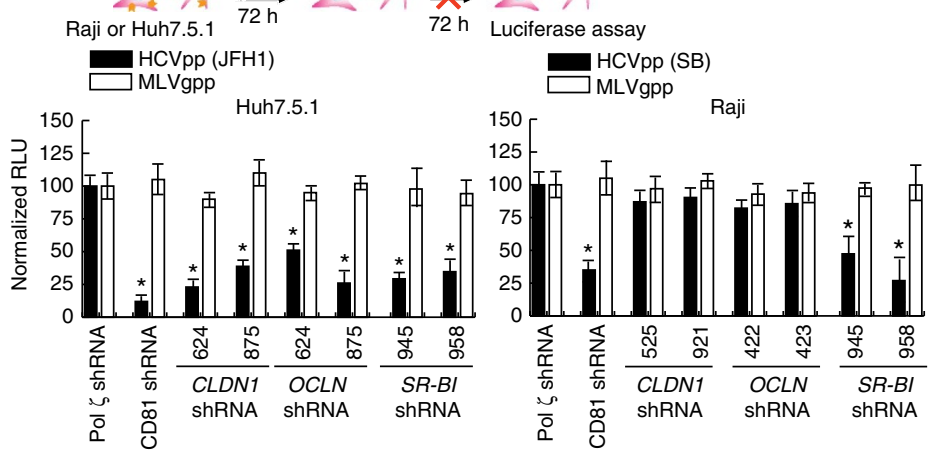

Figure 4 | HCV pseudo-particles (HCVpp) with envelope proteins of SB strain infected B lymphocytes, but not hepatic cells. (a) Claudin1 (CLDN1) or Occuludin (OCLN) expression confers weak susceptibility of Raji cells to JFH1 infection (Upper panel). Immunoblot analysis demonstrated that Claudin-1 and Occludin proteins were expressed by transfection of plasmids. (b) The respective expression vectors of CLDN1 and OCLN were transfected into both Raji and Huh7.5.1 cells. Luciferase-encoding pseudo-particles bearing the indicated envelope proteins were used to infect recipient Raji cells transfected with control vector (white), CLDN1-expressing vector (grey), or OCLN-expressing vector, or control Huh7.5.1 cells (black). For HCVpp, the respective E1E2 genes are indicated in parentheses. Luciferase assay was performed at 3 days after transduction ( ${ }^{\star} P<0.05, t$-test). Values are normalized to the RLU background measured in mock-infected cells (mean of $n=3$; error bars, s.d.). RLU: relative luminescence units. (c) The respective expression vectors of CLDN1 and OCLN were transfected and their protein expression levels were confirmed in both Raji and Huh7.5.1 cells. (Left panel) Expression of both CLDN1 and OCLN (tight junction molecules) promoted HCVpp(SB) entry (right panel) ${ }^{\star} P<0.05, t$-test, $n=3$ ). Error bars represent s.d. (d) Cells were transfected to express shRNA against CLDN1 or OCLN and examined for silencing effects by immunoblots. shRNAs against DNA polymerase $\zeta$ (an irrelevant shRNA for HCV entry) were used as the negative and positive controls, respectively. Two different shRNA clones targeting either CLDN1 or OCLN were used. (e) Silencing of tight junction molecules CLDN1 and OCLN did not inhibit HCVpp(SB) entry in lymphocytes while expression of CLDN1 and OCLN is required for the susceptibility to HCVpp(JFH1) in hepatocytes. Cells were challenged with HCVpp (black bars: pseudotyped with SB or JFH1 E1E2) or MLV-Gpp (white bars) encoding a luciferase reporter ( ${ }^{\star} P<0.05, t$-test, $n=3$ ). Error bars represent s.d.

shRNA-transduced Raji cells for HCVpp entry. As a result of this screen and confirmatory knockdown experiments, B7.2 (or CD86) was identified as a potential entry factor. B7.2 did not affect HEK293T susceptibility to HIV-luc particles either lacking envelope proteins $\left(\mathrm{Env}^{-} \mathrm{pp}\right)$ or pseudotyped with unrelated vesicular stomatitis virus $\mathrm{G}$ protein (VSV-Gp); these served as negative and positive infection controls, respectively. Accordingly, HCVpp-E1-E2 (SB strain) infection of Raji cells was inhibited by anti-B7.2 and anti-CD81/anti-SR-BI (known HCV co-receptors), but not by a control isotype antibody (Fig. 5b).

To further determine if B7.2 is required for HCV-SB infection of B cells, Raji cells were infected with lentiviruses expressing shRNAs targeting different regions of B7.2. The B7.2 knockdown results showed that HCVpp-SB infection was markedly reduced in B7.2-silenced cells as compared to cells treated with a scrambled shRNA (Fig. 5c). When using distinct shRNAs of variable potency against B7.2, HCVpp infectivity was inversely correlated to the degree of silencing (Fig. 5d). By comparison, B7.2 knockdown in Huh7.5.1 cells did not inhibit HCVpp (JFH1) infection (Supplementary Fig. 5A). Next, to determine if the entry step of virus infection required B7.2, synchronized infections were performed on Raji or Huh7.5.1 cells in the presence of blocking antibodies (Fig. 5e). The kinetics of inhibition by anti-B7.2 antibody was significantly slower than that for anti-CD81 antibody, with half-maximal inhibition observed at $128 \mathrm{~min}$ post-temperature shift while the half-maximal inhibition time of anti-CD81 was $32 \mathrm{~min}$. In contrast, anti-B7.2 antibody did not inhibit HCVpp (JFH1) infection of Huh7.5.1 cells. The time course of inhibition was similar to that observed for the Claudin1 antibody $^{16}$. Thus, these results were consistent with the interpretation that anti-B7.2 antibody inhibited a step after virus binding.

We next examined which domains of B7.2 are responsible for interaction with SB envelope proteins. While exogenous B7.2 allowed HCVpp to infect B7.2-silenced Raji cells, neither B7.1 nor ICAM supported HCVpp infection (Fig. 5f). Furthermore replacement of the $\mathrm{IgV}$ domain of B7.2 with that from B7.1 abrogated the HCVpp infection of B7.2-silenced Raji cells (Fig. 5f). The importance of the B7.2 cytoplasmic domain was underscored since replacement of this domain with that from CD8 also resulted in the loss of HCVpp infectivity. To test if a direct interaction occurred between E2 and B7.2, we performed 
a

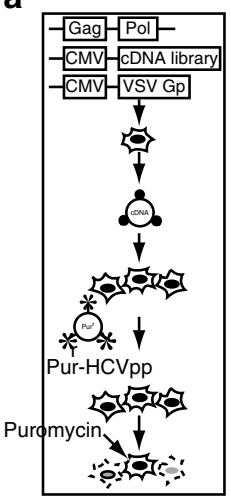

b

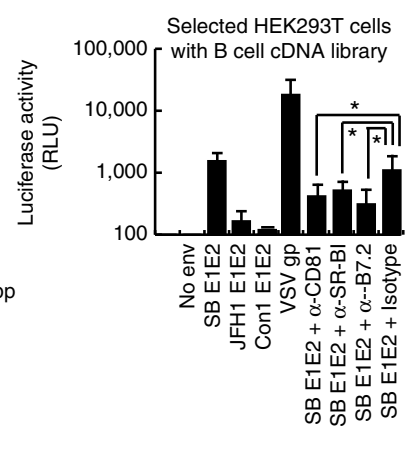

C

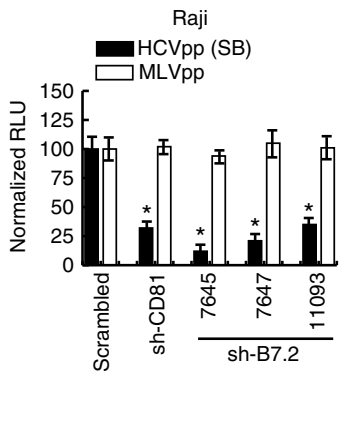

d

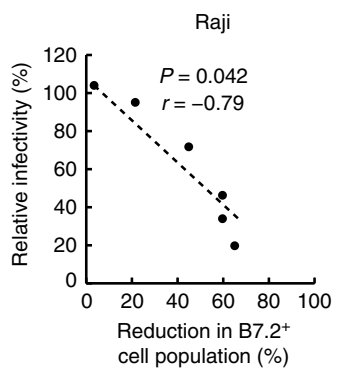

e

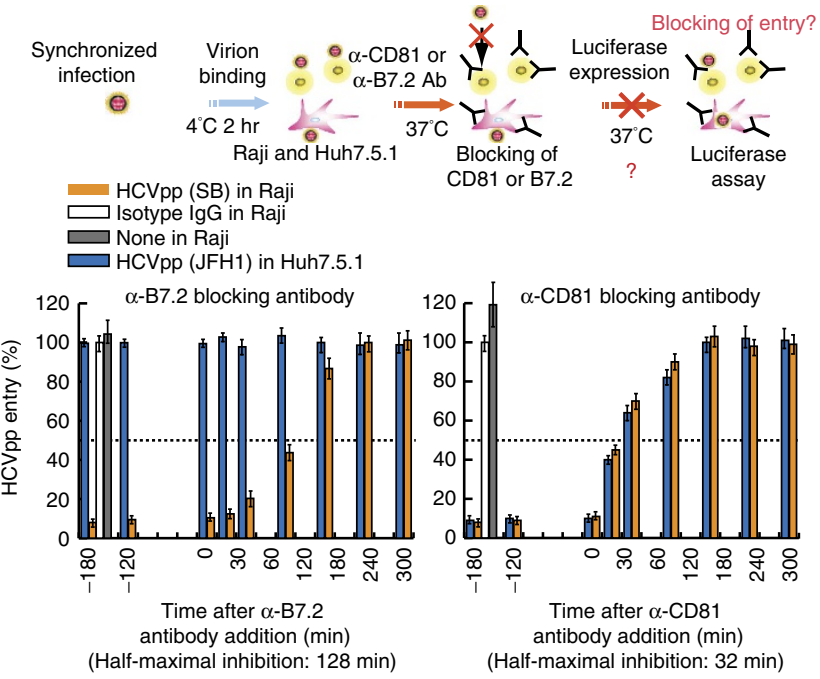

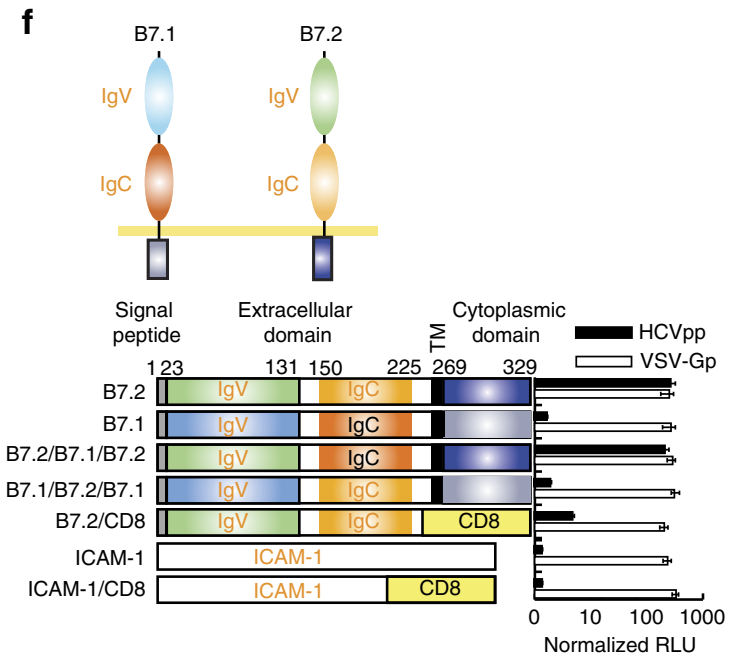

Figure 5 | Co-stimulatory molecule B7.2/CD86 is identified as a co-receptor for lymphotropic HCV infection in B lymphocytes. (a) Expression cloning of lymphotropic HCV receptor. HEK293T cells were transfected with B-cell cDNA library and selected with functional screening using HCVpp(SB) as the ligand and puromycin or other selection antibiotic. To recover B-cell cDNAs in selected (surviving) colonies, DNA was isolated from these selected HEK293T cells and amplified by PCR with specific primers to identify the inserted cDNA library sequence. (b) The blocking effects of anti-B7.2 and anti-CD81 on HCVpp(SB) infection ( ${ }^{\star} P<0.05$, $t$-test, $n=3$ ). Error bars represent s.d. (c) B7.2 silencing inhibited HCVpp-SB entry into Raji cells. The HCVpp entry experiment was performed in triplicate $(n=3)$. Error bars represent s.d. (d) Correlation plot between B7.2 levels and relative infectivity. (e) Time course of the blocking effects of anti-B7.2 and anti-CD81 on HCVpp(SB) infection. Synchronized infections were performed on Raji or Huh7.5.1 cells. Values (percent entry) are relative to the signal seen when the antibody was added at $4 \mathrm{~h}$ post temperature shift. Cellular HCV RNA levels were detected in Raji cell cultures treated with isotype-matched or anti-B7.2 or anti-CD81 antibody. Fits of $t=0$ and later data points represent a one-phase exponential association and sigmoidal dose-response (variable slope) for anti-B7.2 (50 $\left.\mu \mathrm{g} \mathrm{ml}^{-1}\right)$ or anti-CD81 $\left(50 \mu \mathrm{g} \mathrm{ml}{ }^{-1}\right)$ antibody (mean of $n=3$; error bars, s.d.). (f) Both B7.2 lgV and cytoplasmic regions are required for efficient HCV entry. The Raji cells (B7.2 low) transduced with lentivirus expressing sh-B7.2 were electroporated with GFP-B7.2 (B7.2), GFP-B7.2/CD8 (B7.2/CD8), GFP-ICAM-1 (ICAM-1) or GFP-ICAM-1/CD8 (ICAM-1/CD8) vectors. HCV entry levels were assessed for luciferase activities at $72 \mathrm{~h}$ after HCVpp challenge of transfected cells $(n=3)$. Error bars represent s.d.

coimmunoprecipitation with anti-B7.2, followed by immunoblot for E2 proteins and showed an interaction between B7.2 and HCV E2 proteins (Fig. 6a and Supplementary Fig. 9). Reciprocal immunochemical analysis further confirmed E2-B7.2 interactions (Supplementary Fig. 5C and Supplementary Fig. 9). These results indicated that B7.2 is a co-receptor for HCV-SB infection of Raji cells.

We further examined whether the level of B7.2 expression in B cells of hepatitis $C$ patients correlated with the extent of HCV infection. Naïve B cells $\left(\mathrm{CD} 19^{+} \mathrm{CD}^{-} 7^{-}\right)$were found to have low levels of B7.2 expression, while memory B cells $\left(\mathrm{CD} 19^{+} \mathrm{CD} 27^{+}\right)$ showed higher expression levels (Fig. 6b), in agreement with previous reports ${ }^{58}$. Interestingly, memory B cells in HCV patientderived PBMCs expressed significantly lower levels of surface B7.2 when compared to those in HCV ( - ) individuals (Fig. 6c). Significantly, HCV RNA levels were two logs higher in memory
B cells than in naïve B cells isolated from HCV patients, which is correlated with increased protein levels of B7.2 (Fig. 6d). These findings were confirmed by examination of 18 different hepatitis $\mathrm{C}$ patients (Fig. 6e). Lower surface B7.2 levels were correlated with lower cellular HCV RNA levels (Supplementary Fig. 5D). Activation of $\mathrm{B}$ cells (CD40L + IL-4 treatment) is known to promote surface B7.2 expression in naïve B cells ${ }^{59}$. Interestingly, activation of $B$ cells resulted in higher levels of cellular HCV RNA 8 days post-HCV (SB/JFH1) infection (Supplementary Fig. 5E). Anti-CD81, anti-SR-BI or anti-E2 neutralizing antibody treatment blocked HCV infection in sh-RIGI-Raji cells (Supplementary Fig. 5F). B7.2 expression in B and T cells and dendritic cells was confirmed by immunoblot (Supplementary Fig. 5G). These results indicated that CD81, SR-BI and E2 are involved in critical steps for lymphotropic HCV entry process into B cells. This conclusion is limited to B cells, but not in T-cell 

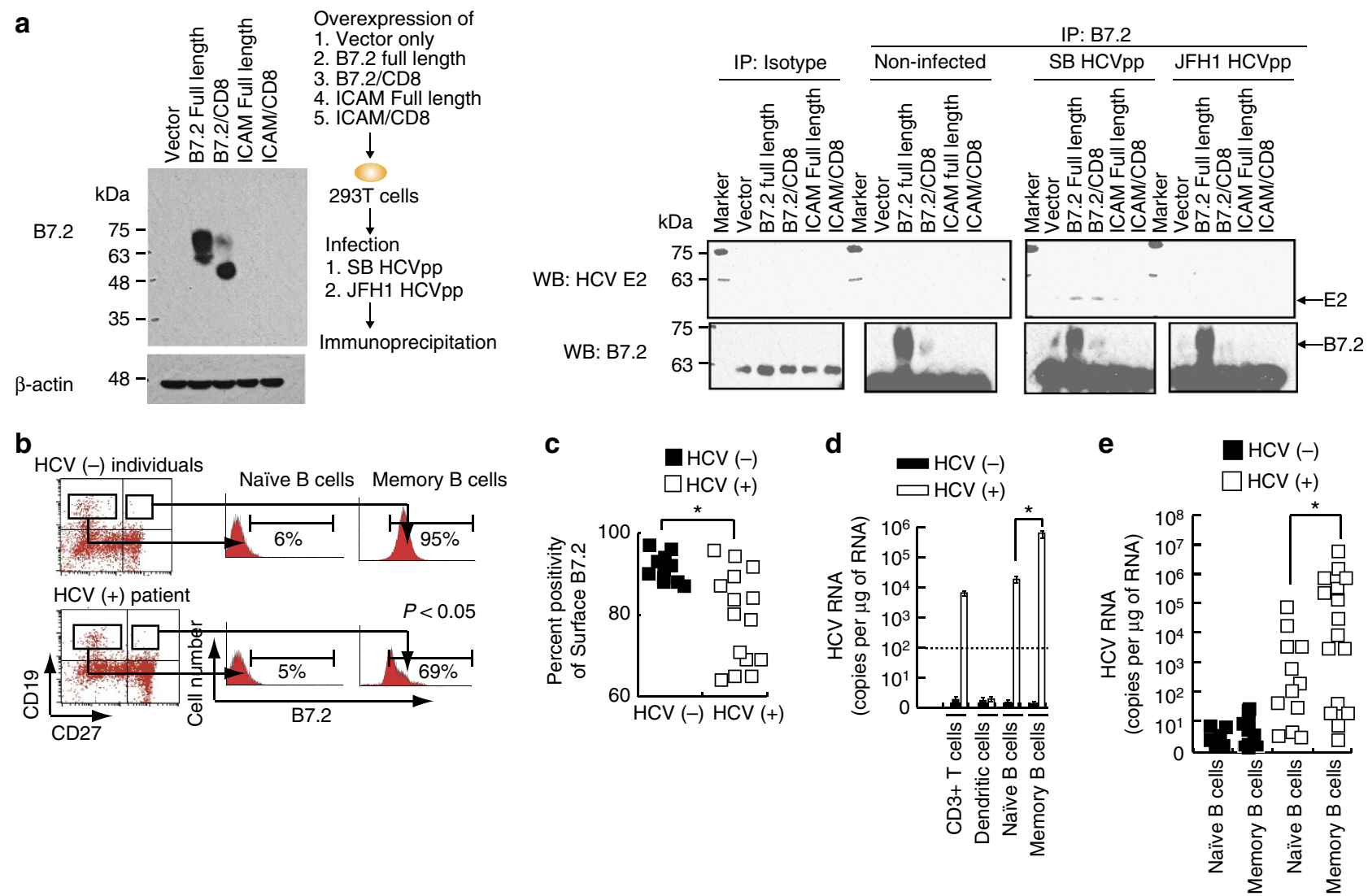

Figure 6 | Envelope protein E2 of HCV SB strain interacts with B7.2 protein in shRIGI-Raji cells and surface B7.2 is reduced in memory B cells of HCV patients. (a) Envelope protein E2 of HCV SB strain interacted with B7.2 protein in shRIGI-Raji cells. Arrows indicate bands of HCV E2. (b) B cells from healthy individuals (upper panel) and HCV patients (lower panel) were sorted by flow cytometry into CD19 + CD27- naïve B cells or CD19 + CD27 + memory B cells. The former expressed a low level of B7.2, whereas the latter expressed a high level of B7.2. (c) Surface B7.2 expression is reduced in human memory B cells in HCV patients. Human patient B cells were sorted into two fractions, naïve B cells $(C D 19+/ C D 27-)$ and memory $B$ $(C D 19+/ C D 27+)$, and used for RNA isolation. (d,e) Memory B cells were infected with HCV. Human memory B cells showed higher levels of HCV RNA in clinical specimens $\left({ }^{\star} P<0.05, t\right.$-test, $\left.n=3\right)$. Error bars represent s.d. Analysis of HCV RNA in B cells, T cells, monocytes and dendritic cells.

lines since SR-B1 involvement is known for HCV infection of primary $\mathrm{T}$ cells. Interestingly, primary $\mathrm{T}$ lymphocytes do not display detectable levels of SR-B1 protein, although T-cell lines do ${ }^{60}$.

Characterization of $\mathrm{HCV}$ particles produced from SB strain. The concentrated virus particles had a density of $1.13 \mathrm{~g} \mathrm{ml}^{-1}$ in sucrose (Fig. 7a), which is in agreement with previous report $\left(1.12 \mathrm{~g} \mathrm{ml}^{-1}\right)$ of $\mathrm{HCV}$ virions isolated from the human Tand B-cell lines HPBMa10-2 and Daudi ${ }^{25}$, but slightly less than that of $\mathrm{HCV}$ virions isolated from hepatocytes (ca. $1.15 \mathrm{~g} \mathrm{ml}^{-1}$ ) (refs 41,61). To further characterize SB viral RNA, SB-transfected cells were metabolically labelled with $\left[{ }^{3} \mathrm{H}\right]$ uridine in the presence of actinomycin D. The viral particles produced from transfected cells were concentrated from the culture supernatant and separated by sucrose gradient sedimentation. A specific ${ }^{3} \mathrm{H}$-uridine-labelled peak, which was resistant to protease treatment, was detected in the culture supernatant of Raji cells (Fig. 7a). RNA isolated from this material yielded a major RNA species of approximately $9.7 \mathrm{~kb}$ and a minor RNA species. The latter could be defective interfering RNA (DI RNA), although further work will be needed to ascertain this (Fig. 7b).

Electron microscopy demonstrated that the particles were approximately $50-60 \mathrm{~nm}$ in diameter and possessed an envelope that was immunoreactive with anti-E2 antibodies (Fig. 7c). Furthermore, ${ }^{35} \mathrm{~S}$-amino acid-labelled virus particles at the peak fraction contained several viral proteins, corresponding to core, E1, and E2, all of which are associated with HCV particles (Supplementary Fig. 6A). The putative identities of ${ }^{35} \mathrm{~S}$-labelled core and envelope protein E2 were confirmed by immunoprecipitation, followed by autoradiography (Supplementary Fig. 6B). It was notable that the virion contained relatively more envelope proteins than core proteins. Treatment with NP-40 disrupted the virus particles, consistent with its identity as an enveloped virion (Supplementary Fig. 6C). The sum of these analyses confirmed that the viral particles produced from the SB-transfected cells in this system had outer cell surface markers consistent with authentic HCV particles.

To determine whether the viral particles released were infectious, we harvested the culture media from SB RNA-transfected Raji cells (sh-RIGI-Raji) which in turn was used to infect naïve Raji cells. The HCV RNA isolated from infected cells was then analysed 8 days later. UV irradiation of virus-containing media prior to infection prevented viral RNA production (Fig. 7d). Also, pretreatment of the transfected cells with IFN- $\alpha$ or $-\gamma$, which inhibits viral RNA replication but not viral attachment, lowered HCV RNA levels in a dose-dependent manner (Fig. 7d). Finally, treatment with Telaprevir ${ }^{62}$, an NS3 (viral protease) inhibitor, or $2^{\prime}$-modified nucleosides as inhibitors of $\mathrm{HCV}$ replication ( $2^{\prime}-\mathrm{C}$-methy adenosine) also reduced $\mathrm{HCV}$ RNA levels in a dose-dependent manner (Fig. 7e).

Active viral RNA replication was also confirmed by the detection of $(-)$ strand RNA in SB-transfected but not 
a

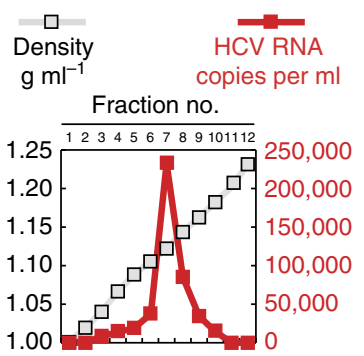

b

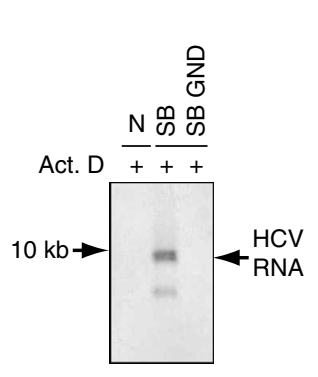

C

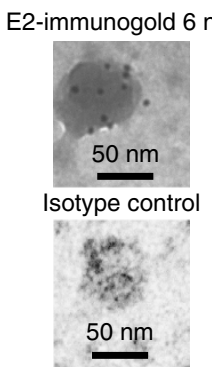

d

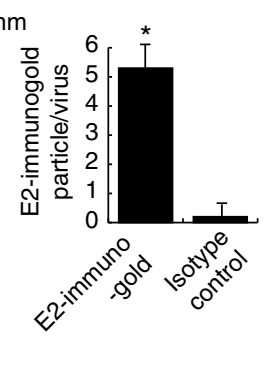

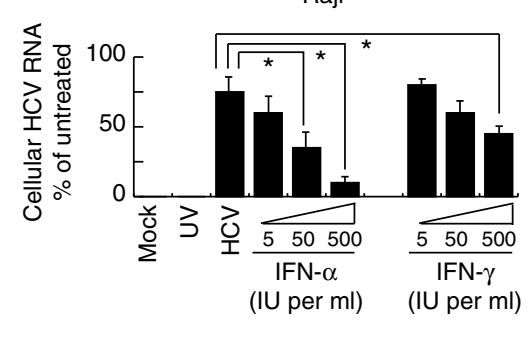

e

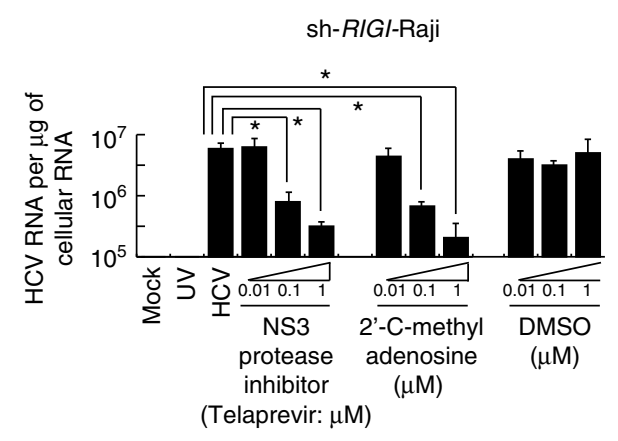

f

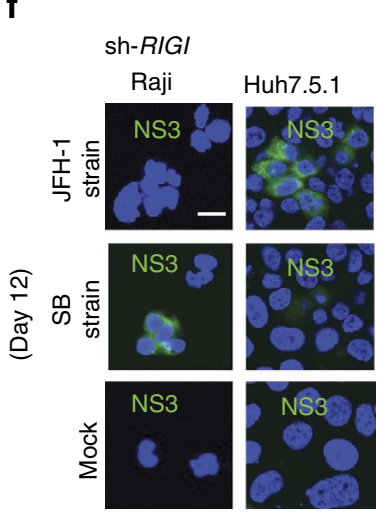

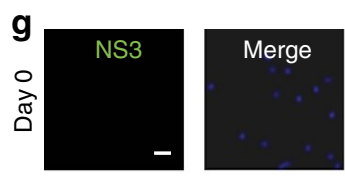

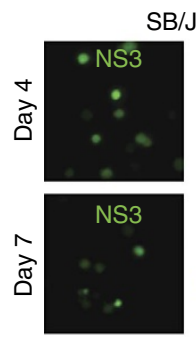

$\mathrm{B} / \mathrm{JFH} 1$
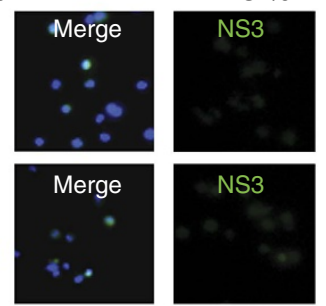

$\mathrm{SB} / \mathrm{JFH} 1$ (GND)
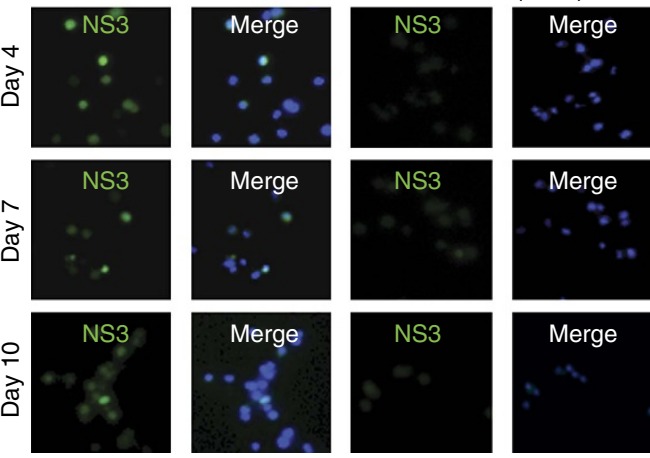

Merge

Figure 7 | The two HCV strains SB and JFH-1 have profoundly different tissue-specificity. (a) Sucrose gradient sedimentation of the supernatant from the transfected cells. Concentrated culture medium collected from SB/JFH1 chimera JFH (SB5'-UTR-NS2) RNA-transfected cells was fractionated using a 20-60\% sucrose density gradient. HCV RNA titer in each fraction was determined by RT-PCR. (b) JFH (SB5'-UTR-NS2)-transfected Raji cells were labelled with $\left[{ }^{3} \mathrm{H}\right]$ uridine for $16 \mathrm{~h}$ in the presence of actinomycin $\mathrm{D}$. The supernatant RNA was concentrated and analysed by denaturing agarose gel electrophoresis. Radiolabelled RNAs were visualized with a BAS-2500 Bio-Imager (Fuji). (c) Electron micrograph of the SB virus concentrated by sucrose gradient fractionation. The virus was stained with anti-E2 or isotype antibody and Protein A-conjugated immunogold. Asterisk represents statistical significance $(P<0.05, t$-test, $n=3)$. Error bars represent s.d. (d) Inhibition of HCV infection by IFN. Raji cells were treated with 5,50 and 500 units ml ${ }^{-1}$ human IFN- $\alpha$ and IFN- $\gamma$ (PBL Biomedical Laboratory, Piscataway, NJ, USA) for $6 \mathrm{~h}$ and then inoculated with SB virus in the presence of the same doses of IFN. The viral inoculum was removed $4 \mathrm{~h}$ later, and the cells were further cultured with IFN for 8 days. Cellular HCV RNA was isolated and analysed by real-time RT-PCR $\left({ }^{\star} P<0.05, t\right.$-test, $\left.n=3\right)$. Error bars represent s.d. (e) SB-infected Raji cells (sh-RIGI-Raji) were treated with an NS3 protease inhibitor (Telaprevir) or $2^{\prime}$-modified nucleosides as inhibitors of HCV replication ( $2^{\prime}-\mathrm{C}$-methy adenosine) ( ${ }^{\star} P<0.05, t$-test, $n=3$ ). Error bars represent s.d. (f) Immunofluorescence of NS3 in cells infected with SB or JFH1 RNA. Staining was done at 12 days post-infection. Green (NS3), Blue: DAPI staining. Scale bar represents $10 \mu \mathrm{m}$. (g) Time course of NS3-positive B cells after HCV infection. Scale bar represents $10 \mu \mathrm{m}$.

JFH1-transfected sh-RIGI-Raji cells (Supplementary Fig. 6D). Addition of anti-E2 neutralizing antibody prevented ( - ) strand RNA synthesis whereas control, non-specific isotype antibody treatment had no effect on HCV replication (Supplementary Fig. 6D, Lower panel). A quantitative estimate of $(+)$ and $(-)$ strand HCV RNA synthesis showed the former was tenfold higher in abundance than $(-)$ genomic RNA. These results clearly demonstrated that active SB HCV RNA replicates in B cells.

By immunofluorescence studies, we also showed that NS3positive cells could be detected in lymphotropic SB-transfected Raji cells, but not in Huh7.5.1 cells. In contrast, NS3 was detected in hepatotropic JFH-1-transfected Huh7.5.1 cells, but not Raji cells (Fig. 7f). Transfection of replication incompetent strain $\mathrm{SB} / \mathrm{JFH} 1$ (GND) led to significantly lower levels of NS3 staining at days 7 and 10 post-transfection (Fig. $7 \mathrm{~g}$ ). As a specificity control, SB chimera containing NS5B GND mutations, which cannot replicate in Raji cells (Construct G), did not yield any HCV RNA. These results indicated that the RNA signal was indeed due to viral infection and replication and not due to trapping of viral particles on the outer cell surface. This finding was confirmed by immunoblot (Supplementary Fig. 6E). Taken together, these results demonstrated that $\mathrm{HCV} \mathrm{SB}$ preferentially infects, replicates and produces infectious virions in sh-RIGI-Raji cells, whereas JFH-1 preferentially infects and replicates in Huh7.5.1 cells.

Persistent HCV infection of PBMC in a humanized mouse model. We extended our study of the lymphotropic properties of HCV-SB in vivo by using humanized mice. For this purpose we generated mice with stably engrafted human CD34+ hematopoietic progenitor cells into an immune-compromised background line [Rag2-/-;Il2rg-/- (RG)-hu HSC mice]. Analysis of these animals (RG-hu HSC mice) following engraftment indicated that $6-37 \%$ of immune cells were positive for $\mathrm{CD} 45+$ of human origin that was maintained through 10 weeks post-engraftment. After human peripheral blood leukocytes 
Table 1 | HCV infection analysis on immunocompromised mice transplanted with human hematopoietic stem cells (HSCs).

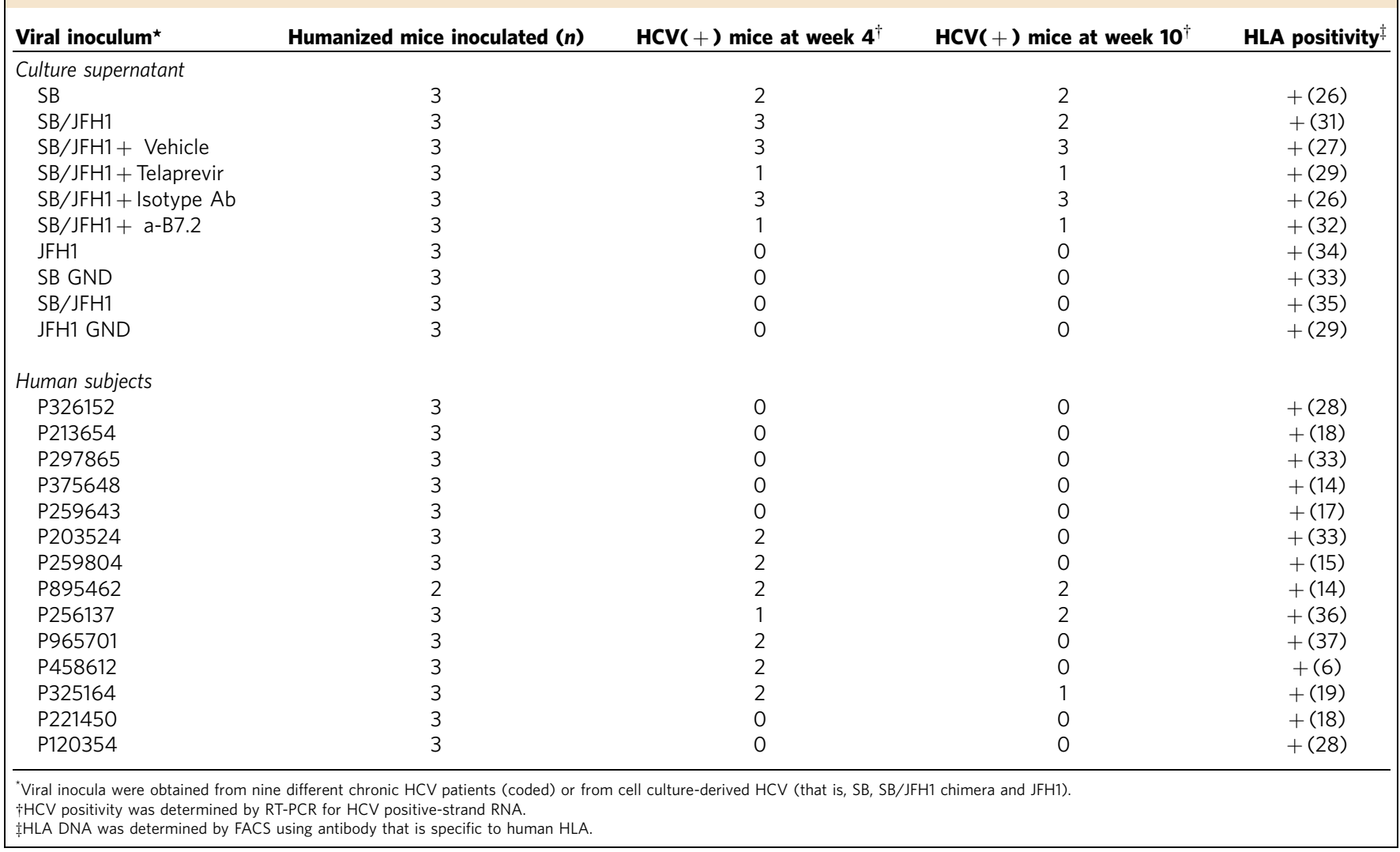

a

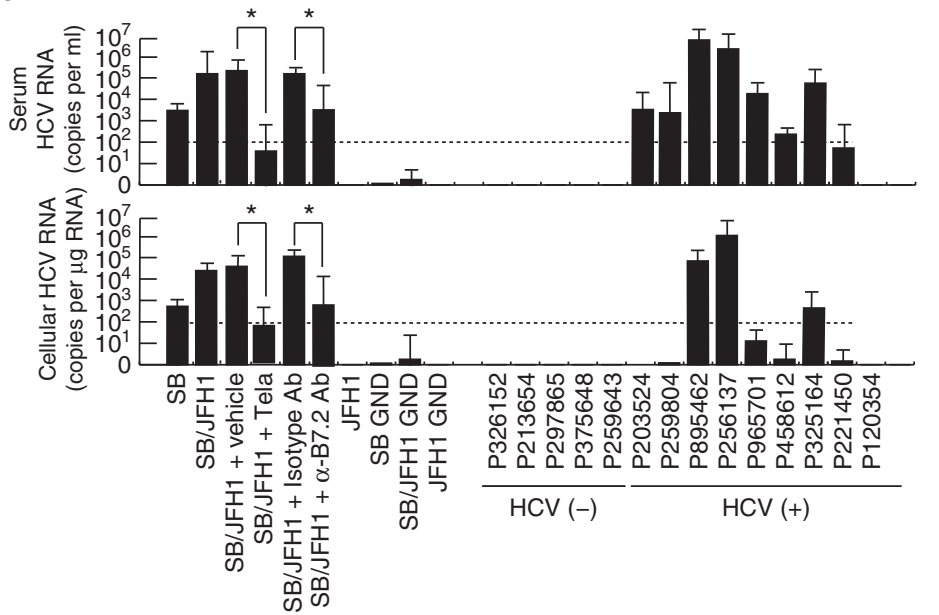

b

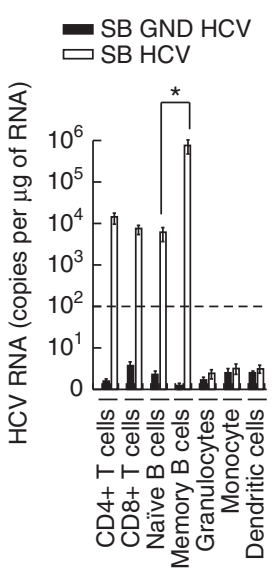

Figure 8 | A humanized mouse model engrafted with human hematopoietic cells was utilized to show HCV infection of human lymphomononuclear cells in vivo by the lymphotropic HCV strain. (a) HCV infection in immunocompromised Rag2;/l2rg - / - (RG) mice engrafted with human hematopoietic stem cells (RG-hu HSC Mice) shows that the two HCV strains, SB and JFH-1, have different tissue-specificity. HCV RNA levels in serum and PBMCs isolated from RG-hu HSC mice at 70 days after HCV inoculation were determined by RT-qPCR. Tela: telaprevir (HCV NS3 inhibitor) treatment. $\left({ }^{\star} P<0.05, t\right.$-test, $\left.n=3\right)$. Error bars represent s.d. (b) Human PBMCs from the infected RG-hu mice collected at 70 days after HCV inoculation were separated into various cell types and analysed for the presence of HCV RNA Memory B cells have high cellular HCV RNA levels in comparison to that of naïve B cells. ( ${ }^{\star} P<0.05, t$-test. $n=3$ ). Error bars represent s.d.

were detected at 16 weeks post-engraftment, the mice were intravenously injected with either cell culture-derived HCV-SB virus, $\mathrm{HCV}-\mathrm{JFH} 1$ virus or a chimeric $\mathrm{SB} / \mathrm{JFH} 1$ virus, the latter of which contains the SB 5'-UTR-NS2 genes of the JFH1 genomic backbone (construct F). The HCV RNA levels in the PBMC of these mice were then analysed by RT-PCR at different time points after infection. As shown in Table 1, five of the six mice infected with either SB or SB-JFH1 chimeric virus tested positive for HCV RNA at 4 weeks post-inoculation; four of these six mice remained HCV RNA positive for more than 10 weeks after 
virus injection. HCV RNA was detected in PBMCs of these mice (up to $2.7 \times 10^{5}$ particles $\mathrm{ml}^{-1}$ : Fig. $8 \mathrm{a}$ ). In contrast, mice injected with JFH1 virus or the replication-incompetent strains (GND mutant) of SB or JFH1 strains were negative for HCV RNA.

We infected humanized mice with sera from 14 human subjects, nine of whom tested positive for HCV. None of the $\mathrm{HCV}$-negative sera led to infection in these mice (Table 1). In contrast, 13 of 24 mice inoculated with the HCV-positive sera tested positive for HCV RNA at week 4, of which five mice remained positive up to week 10. Injection of anti-B7.2 antibody reduced both internal and cell surface associated HCV RNA levels (Fig. 8a). Human PBMC from the infected Rag2 - / - ;Il2rg - /(RG) mice were further sorted into $\mathrm{T}$ and $\mathrm{B}$ lymphocytes, monocytes, and dendritic cells and examined separately for cellular HCV RNA. As shown in Fig. 8b, the HCV RNA was preferentially detected in memory B cells. HCV RNA was also detected in CD4 + and CD8 $+\mathrm{T}$ cells in humanized mice (Fig. 8b). These results indicated that $\mathrm{HCV}$ was capable of persistently infecting human B cells in a humanized mouse model.

HCV binding downregulates B7.2 and inhibits Ig production. As $\mathrm{CD} 27+$ memory $\mathrm{B}$ cells in $\mathrm{HCV}$ patients are anergic (exhausted) B cells ${ }^{63,64}$, the functions of memory B cells infected with HCV particles were examined accordingly. To determine if $\mathrm{HCV}$-infected $\mathrm{CD} 27+\mathrm{B}$ cells had attenuated signalling upon BCR ligation, $\mathrm{Ca}^{2}+$ mobilization was measured after immunoglobulin (Ig)M stimulation with anti-human IgM. The $\mathrm{HCV}$-infected $\mathrm{CD} 27+\mathrm{B}$ cells had diminished $\mathrm{Ca}^{2+}$ mobilization (Fig. 9a), indicating that $\mathrm{HCV}$-infected $\mathrm{CD} 27+$ $\mathrm{B}$ cells are anergic to BCR-mediated stimulation. To test if HCV-infected cells were prone to apoptosis, HCV-infected primary memory B cells isolated from patients were stimulated with CD40L + IL2 + IL-10 or vehicle control ex vivo (Supplementary Fig. 9A). Treated memory B cells were stained with annexin $\mathrm{V}$ (to measure apoptosis) and anti-CD27. After incubation with vehicle control or CD40L + IL2 + IL-10 treatment, the percentage of annexin $\mathrm{V}^{+}$cells was higher in the CD40L + IL4-stimulated HCV-infected B-cell subset than that of UV-irradiated supernatant-treated cells (Supplementary Fig. 9A). As CD40L stimulation transactivates pro-apoptotic molecule Bax (ref. 65), Bax mRNA levels were also examined. The treatment was found to induce BAX after cross-linking B7.2 on HCV-infected memory B cells (Supplementary Fig. 9A, right). These data suggested that, in the absence of survival signals, $\mathrm{HCV}$-infected CD27 + B cells were prone to apoptosis.

B7.2 is a costimulatory molecule that interacts with CD28 and CTLA-4 on $\mathrm{T}$ cells. In addition, B7.2 has a cytoplasmic tail that transduces signals in antigen-presenting cells ${ }^{66,67}$. B7.2 stimulation results in the phosphorylation of phospholipase $\mathrm{C} \gamma 2$ (PLC $\gamma 2$ ) and protein kinase $\mathrm{C} \alpha \beta(\mathrm{PKC} \alpha / \beta)$, leading to activation of transcription factor Oct- 2 and eventual increased IgG1 transcription and protein production ${ }^{68}$. Since binding of the HCV E2 protein to CD81 can perturb the biological activities of CD81 (ref. 69), we tested if the binding of HCV E2 to B7.2 also affected B7.2 and its signalling pathways. To determine whether HCVpp-SB infection reduced CD28/Fc-mediated activation of PLC $\gamma 2$ and PKC $\alpha / \beta$, we analysed the activation of PLC $\gamma 2$ and $\mathrm{PKC} \alpha / \beta$. As shown in Supplementary Fig. 9B, CD28/Fc-mediated phosphorylation of PLC $\gamma 2\left(\mathrm{Tyr}^{1217}\right)$ and $\mathrm{PKC} \alpha / \beta\left(\mathrm{Thr}^{638 / 641}\right)$ was reduced in HCVpp-infected memory B cells.

To test if the observed effects on signalling were due to the E2-B7.2 interaction, we used purified recombinant E2 (SB strain) purified from Baculovirus expression system instead of HCVpp-SB. To test if E2 (SB strain) binds to B7.2, His-tagged E2 (without the transmembrane domain) from SB strain (genotype 2b) and H77 strain (genotype 1a) were expressed in the baculovirus expression system and partially purified (Supplementary Fig. 7A). The intracellular E2 was detected in both monomeric and aggregated forms (Supplementary Fig. 7B). Immunoblot analysis confirmed expression of HCV E2 protein (Supplementary Fig. 7C). The partially purified E2 was used for in vitro binding to various cell lines as determined by flow cytometry. The results showed that E2 from the SB strain bound to Raji cells very efficiently. E2 from the genotype 2a JFH1 strain does not bind to HepG2 cells (Supplementary Fig. 7D). The binding strengths of E2 from SB and JFH1 strains were further compared by using different amounts of E2 (normalized using an anti-His $_{6}$ antibody). E2 did not bind to HepG2 cells, which is among the few human cell lines that do not express CD81 or B7.2. In contrast, E2 bound to the B7.2-overexpressing HepG2 cells efficiently (Supplementary Fig. 7D). The binding was partially blocked by a B7.2-specific antibody but not by an isotype-matched control antibody (Supplementary Fig. 7D). As shown in Supplementary Fig. 7E, E2 of the SB strain bound more than that of the H77 strain at every protein concentration used. These results indicated that recombinant E2 (SB lymphotropic strain), but not E2 (H77 hepatotropic strain), bound to B cells in a B7.2-dependent manner.

Furthermore, to rule out that the observed effects were not a consequence of CD81 engagement by E2-SB, endogenous CD81 was knocked out by CRISPR and replaced with exogenous full-length CD81 or extracellular-domain-truncated CD81 expression vectors. CD81 overexpression partially restored immunoglobulin production in CRISPR-mediated CD81 knockout cells (Supplementary Fig. 8A). To further test if another co-receptor SR-BI stimulated immunoglobulin production, SR-BI was knocked out by CRISPR and replaced with transfected full-length SR-BI or intracellular-domain-truncated mutant of SR-BI and examined for immunoglobulin production. Both C-terminus truncation mutant and full-length SR-BI showed no effect on IgM production in the CRISPR SR-BI knockout cells expressing C-terminus truncation mutant of SR-BI or full-length SR-BI (Supplementary Fig. 8B). B2.7 restoration promoted IgM production while $\mathrm{B} 7.2 / \mathrm{CD} 8$ chimera, which lacks the signal transduction domain, failed to restore the IgM production (Supplementary Fig. 8C, left). These results indicated that HCV E2(SB)-mediated downregulation of B7.2 reduced CD28/Fc-mediated signalling pathways.

HCV-infected B cells do not differentiate into plasmablasts. To test whether binding of HCV to B7.2 inhibited B7.2-induced signalling pathways, resting $\mathrm{B}$ cells were purified from human PBMC for challenge with HCVpp-SB. As B7.2 signalling promotes the IgM production, supernatants were collected on day 6 following the administration of CD40L + IL-2 + IL-10 and assayed by enzyme-linked immunosorbent assay (IgM ELISPOT assay) for IgM production from these cells. Memory B cells cultured with medium alone or with control antibody did not induce antibody secretion. As illustrated in Fig. 9b, memory $\mathrm{CD} 27^{+} \mathrm{B}$ cells were infected with HCVpp and stimulated with CD40L, IL-2 and IL-10 for 6 days, which differentiated B cells into antibody-secreting cells. Similarly, treatment with CD28/Fc fusion protein induces B7.2 signalling and activates Ig production pathways ${ }^{68}$. The pseudotyped MLV viral particle, which does not bind to B7.2 but infects B cells, did not affect CD28-mediated IgM production (Fig. 9b). We observed that the HCVpp-infected $\mathrm{CD} 27^{+} \mathrm{B}$ cells were less efficient at differentiating into IgM-secreting plasmablasts 
a

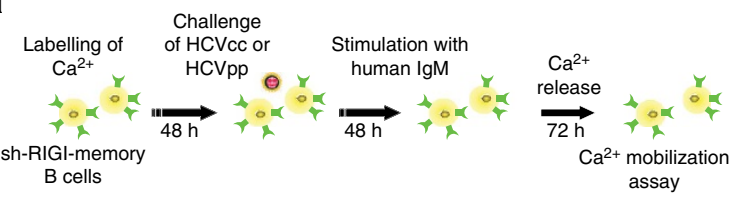

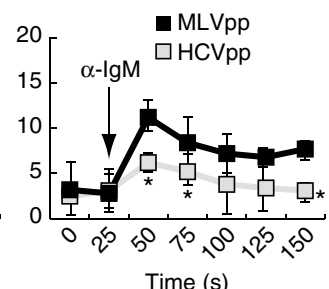

b
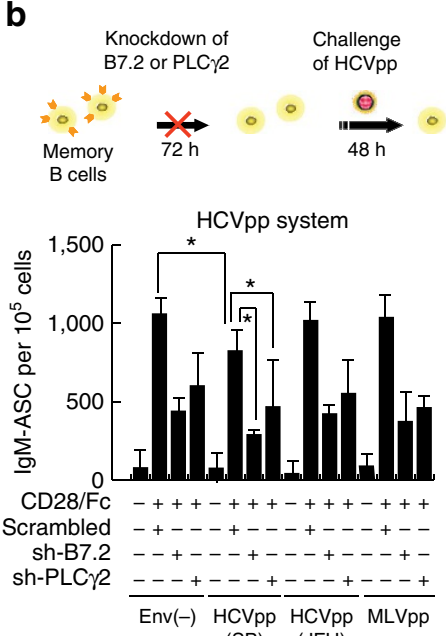

(SB) (JFH)

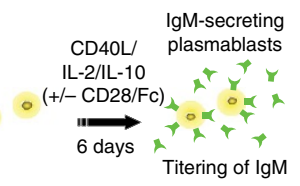

HCVpp system

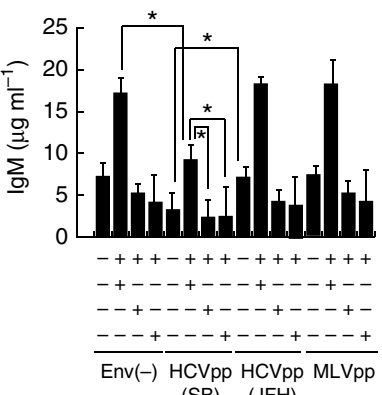

(SB) (JFH)

c
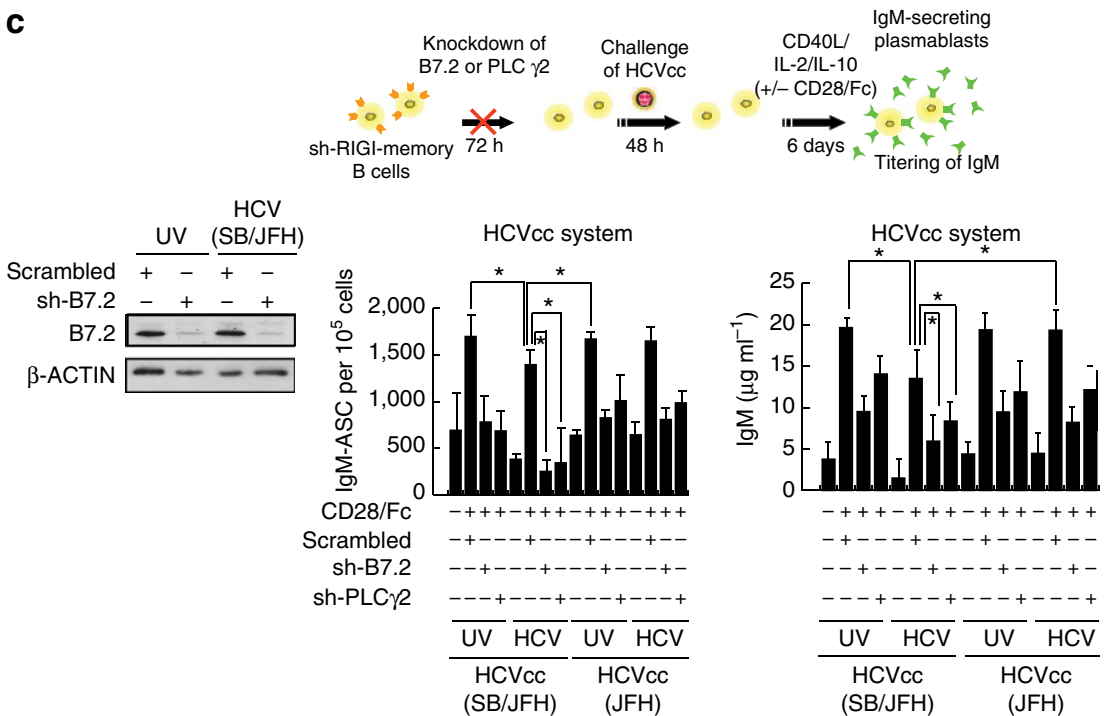

d
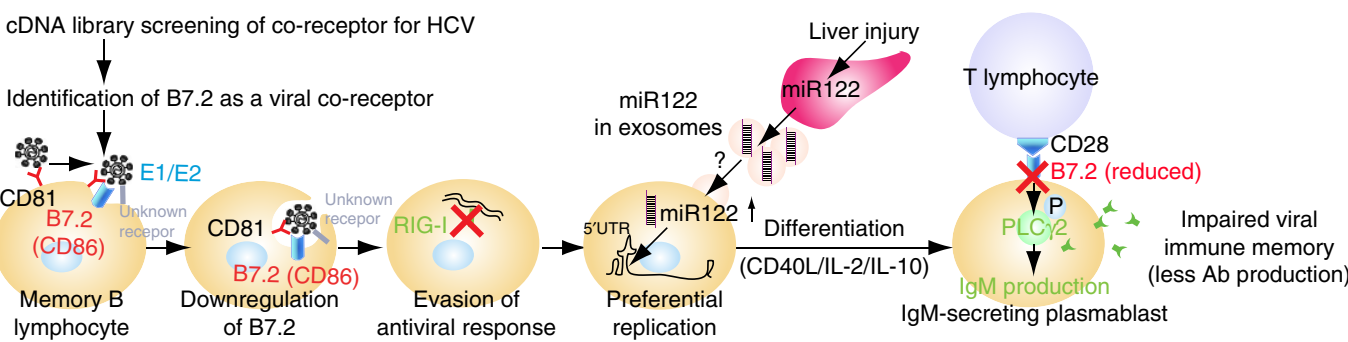

Figure 9 | HCV infection impairs antigen recall responses in memory B cell. (a) HCV-infected CD27 + B cells, compared to uninfected CD27 + B cells, have attenuated $\mathrm{Ca}^{2+}$ responses after BCR cross-linking. B cells from healthy donor were loaded with Indo-1-AM, stained with anti-CD27 and warmed to $37^{\circ} \mathrm{C}$. After establishing a baseline for $30 \mathrm{~s}$, cells were stimulated with $10 \mu \mathrm{g} \mathrm{ml} \mathrm{l}^{-1} \mathrm{goat} \mathrm{F}\left(\mathrm{ab}^{\prime}\right) 2$ anti-human IgM. Kinetic graphs represent ratios of bound/ unbound Indo-1 AM over time for CD27 + B-cell populations. Arrows indicate addition time of $F\left(a^{\prime}\right) 2 \alpha$-human $\lg M\left({ }^{\star} P<0.05, t\right.$-test, $\left.n=3\right)$. Error bars represent s.d. (b) Signals by anti-B7.2 Ab induce the production of IgM by memory B cells. Memory B cells $\left(5 \times 10^{3}\right.$ cells per well) were cultured with different concentrations of anti-B7.2 $\left(0.5 \mu \mathrm{g} \mathrm{ml}^{-1}\right)$; after 5 days, supernatants from control and experimental wells were collected and lgM levels were measured. Data are presented as the fold-increase above memory B cells activated with a CD4OL + IL-2 + IL-10, and a species- and isotype-matched control $\mathrm{Ab}\left({ }^{\star} P<0.05, t\right.$-test, $\left.n=3\right)$. Error bars represent s.d. (c) Similar assays were performed using HCVcc as described with $H C V p p$ above $\left({ }^{\star} P<0.05\right.$, $t$-test, $n=3$ ). Error bars represent s.d. (c, inset) Silencing effect of shRNA targeting B7.2 was confirmed by immunoblot. (d) A model of lymphotropic HCV infection in memory B cells. B7.2 serves as one of the co-receptors for HCV-SB infection of B cells. B7.2 (CD86) mediates lymphotropism of HCV infectious clone derived from HCV SB strain towards human memory B cells and its binding suppresses the cells' function by downregulating surface expression of B7.2. Evasion of antiviral responses (that is, RIGI), induction of miR-122, and another host factor targeting HCV 5'-UTR are involved in stimulating HCV replication in B cells. HCV-mediated downregulation of B7.2 impairs anti-viral immune memory, including reduction of antibody production and antigen recall responses. 
following CD40L/IL-2/IL-10 stimulation (Fig. 9b, left). This suggested differentiation to IgM-secreting plasmablasts upon CD40L/IL-2/IL-10 stimulation was impaired in HCVppinfected memory B cells, compared to uninfected memory B-cells (Fig. 9b, left). These results indicated that HCVpp-SB-mediated downregulation of B7.2 reduced $\mathrm{CD} 28 / \mathrm{Fc}$-mediated signalling pathways. Silencing B7.2 or PLC $\gamma 2$ significantly reduced IgM production in HCV-infected memory B cells (Fig. 9b, right). In contrast, HCVpp-JFH1, which does not bind to B7.2, did not inhibit IgM production. To confirm the biological relevance of the pseudotyped HCV particle study, similar results were also obtained when we used the HCV-SB virus (Fig. 9c). Class switch did not account for the decreased IgM in these subsets, as we consistently detected $<140 \mathrm{ng} \mathrm{ml}^{-1}$ of IgM in cell culture supernatants. Taken together, these results indicated that infection with both $\mathrm{HCV} c \mathrm{cc}$ and $\mathrm{HCVpp}$ increased IgM production and by downregulation of B7.2, attenuated CD28-mediated IgM production; thus virus infection enhanced the differentiation of memory $\mathrm{B}$ cells into IgM-secreting plasmablasts.

\section{Discussion}

In this study, we have established the genetic basis for lymphotropism of HCV infection. A comprehensive set of data indicated that B7.2 is a co-receptor for observed HCV SB strain tropism towards memory B cells. Previous reports have also indicated that some HCV strains can infect human T cells: the $\mathrm{H} 77$ strain of HCV can infect and replicate in established T-cell lines, such as Molt- 4 and Jurkat cells, in long-term culture ${ }^{21}$. Furthermore, transfection of SB strain RNA, but not JFH1, led to its replication in Molt- 4 cells $^{70}$. Here we provide evidence indicating that B7.2 is involved in HCV SB strain tropism towards memory B cells (additional discussion is described in Supplementary Note 1$)$.

B7.2 belongs to the immunoglobulin superfamily, and is highly expressed on memory $\mathrm{B}$ cells and germinal centre $\mathrm{B}$ celll $^{71}$. Its ligand includes CD28 and CTLA4 on the surface of T cells. B7.2 and B7.1 are expressed by antigen-presenting cells including $B$ cells. The result of ligation of B7.2 leads to the following: B7.2-CD28 interaction co-stimulates T-cell activation and enhances T-cell survival, B7.2-CTLA4 interaction inhibits T-cell activation and leads to T-cell tolerance. The infection of $\mathrm{B}$ cells by HCV likely causes significant alteration of these functions ${ }^{72}$. Furthermore, expression of HCV viral proteins in B cells can result in B-cell lymphoma in transgenic mice ${ }^{73}$. The expression levels of B7.2 are not correlated with cellular HCV RNA levels in certain primary cells, such as dendritic cells-which are known to be associated with $\mathrm{HCV}^{74}$-indicating that other co-receptor(s) may exist for lymphocyte infection (Supplementary Fig. 5G). Indeed, CD5 expression is linked to HCV infection of lymphocytes ${ }^{75}$. Another possibility is that inhibitors of HCV infection, such as Ewi-2wint, a partner of CD81, may be associated with this lymphotropism ${ }^{76}$. Other studies have shown ApoE to be a tropism determinant in a late assembly step and viral cell-to-cell transmission of HCV JFH1 strain ${ }^{77}$.

In conclusion, $\mathrm{HCV}$ tissue tropism is determined primarily by the properties of viral envelope proteins and the $5^{\prime}$-UTR, in addition to the ability of $\mathrm{HCV}$ to evade antiviral responses (Fig. 7f). The existence of lymphotropic HCV strains indicates that lymphoid cell infection may be an important facet of $\mathrm{HCV}$ infection in some patients. These studies on the genetic basis of lymphotropism of HCV will open up an avenue for studying extrahepatic infection of $\mathrm{HCV}$ in vivo and in vitro. The SB strain may be representative of lymphotropic quasispecies that arise from an HCV infection that is primarily hepatotropic.

\section{Methods}

Cells. Raji (Cat\# CCL-86), HEK293T (Cat\# CRL-3216) and HepG2 (Cat\# HB-8065) cells were obtained from ATCC. HepG2 cells were transfected with pCDNA3.1-CD81 or B7.2 expression plasmid, selected with G418 $\left(0.5 \mu \mathrm{g} \mathrm{ml}^{-1}\right)$ and surviving cell colonies were picked after drug selection. Individual cell clones were further isolated and characterized for CD81 expression and E2 binding. To generate sh-RIGI-Raji cells, Raji cells were transduced by multiple lentivirus expressing shRNAs against RIG-I and MDA5 (Open Biosystems) and/or miR122. The culture media for Raji and sh-RIGI-Raji cells is RPMI media 1640, GlutaMAX (Cat\#61870-036, Life Technologies) containing 20\% FBS (Omega, Inc.). PBMC were isolated by Ficoll-Paque Plus (Amersham Biosciences) density gradient centrifugation. Informed consent was obtained. Institutional Review Board at University of Southern California approved the procedures. PBMC were maintained in RPMI 1640 containing 10\% FBS. Infection of cells, generation of HCV pseudo-particles and generation of HCV clone ar described in Supplementary Methods.

Vector. The Claudin-1 expression vector was a gift from Dr Weeraratna from National Institute of Aging (NIA/NIH). WT B7.2, B7.2-CD8, ICAM-1/CD8 and WT ICAM-1 expression vectors were previously described (gifts from Dr Jung, USC) (ref. 78).

Sucrose density gradient analysis. The viral particles produced from the transfected cells were concentrated from the culture supernatant and separated by sucrose gradient sedimentation. Culture medium derived from sh-RIGI-Raji cells was harvested for sucrose density gradient analysis 6 days after transfection of fulllength SB RNA. Collected culture medium was cleared by low speed centrifugation at $670 \mathrm{~g}$ for $10 \mathrm{~min}$, and passed through a filter with $0.45-\mu \mathrm{m}$ pore size (Millipore). Filtered culture medium was layered on a stepwise sucrose gradient (60 to $10 \%$, $\mathrm{wt} / \mathrm{vol}$ ) and centrifuged for $16 \mathrm{~h}$ in a SW41 rotor (Beckman) at 40,000 or $50,000 \mathrm{rpm}(273,865 g$ or $427,914 g)$ at $4^{\circ} \mathrm{C}$. After centrifugation, 8 or 16 fractions were harvested from the bottoms of the tubes. Core protein concentration in each fraction was determined by an immunoassay using $100 \mu \mathrm{l}$ of the fraction. HCV RNA titer was determined by RTD-PCR using RNA isolated from $100 \mu \mathrm{l}$ of the fraction. The ${ }^{35} \mathrm{~S}$ labelling was performed by standard procedures.

Lentivirus production. To generate RIGI or MDA5 shRNA or a miR-122 lentivirus, the transfer vector expressing $m i R-122$ or shRNA targets sequence on the RIG-I or MDA5 cDNA (RHS4531-NM_022168, RHS4430-98851910, RHS443099158156, RHS4430-99161339: MDA5 so called DDX58, or RHS4531-NM_014314, RHS4430-98910525, RHS4430-99294017, RHS4430-99619590: RIG-I so called DDX58; Human GIPZ Lentiviral shRNAmir target gene set, Open Biosystems) were co-transfected with pPAX2 and pVSV-Gp expression vector in HEK293T cells.

Cell entry assay. Virus infection was carried out at $4{ }^{\circ} \mathrm{C}$ (no endocytosis) and then shifted to $37^{\circ} \mathrm{C}$ (active endocytosis). Antibody-treated and untreated cultures were infected with $\mathrm{HCVpp}(\mathrm{SB})$ or $\mathrm{HCVpp}(\mathrm{JFH} 1)$ for $1 \mathrm{~h}$ at $4^{\circ} \mathrm{C}$. Plates were then brought to $37^{\circ} \mathrm{C}$ and assayed at indicated time points over a $300 \mathrm{~min}$ time course. Controls are normalized to the value for anti-CD81 added at $4 \mathrm{~h}$. Antibodies directed against CD81 (JS81) or B7.2 were added at various time points after temperature was shifted to $37^{\circ} \mathrm{C}$, and the infectivity was determined at 12 days after infection as previously described. The concentration of B7.2 and CD81 we used in the experiments are both $50 \mu \mathrm{g} \mathrm{ml}^{-1}$. Half-maximal inhibition time of anti-B7.2 was $132 \mathrm{~min}$ while half-maximal inhibition time of anti-CD81 was $44 \mathrm{~min}$.

Immunoprecipitation followed by immunoblot for B7.2. Full-length of B7.2, B7.2/CD8, full-length of ICAM or ICAM/CD8 were overexpressed in 293T cells. After $24 \mathrm{~h}$, the expression level of B7.2 was determined by western blots. For infection, the culture media was replaced with fresh media containing either SB HCVpp or JFH1 HCVpp and incubated an additional $24 \mathrm{~h}$. Cell lysates were used for mmunoprecipitation with anti-B7.2, followed by immunoblot for B7.2 proteins in $293 \mathrm{~T}$ cells.

After incubation, the cells were harvested and lysed for immunoprecipitation. B7.2 only interacted with HCV E2 after SB infection, but not JFH1 infection. These data indicate that SB E2 protein, but not JFH1 E2 protein, directly interacted with B7.2.

Electron microscopy. Raji cells were transfected with HCV RNA and cell-free supernatant was harvested $96 \mathrm{~h}$ post transfection and concentrated via ultracentrifugation. Grids were washed on drops of PBS, fixed with 3\% paraformaldehyde in PBS and blocked in a solution of $0.8 \%$ BSA, $1 \%$ cold water fish skin gelatin (Sigma) and $20 \mathrm{mM}$ Glycine in PBS. Immunogold labelling was performed with an antibody directed against E2 (CBH5) diluted 1:50 in blocking solution, and Protein A coupled to $10 \mathrm{~nm}$ gold particles. After extensive washing with PBS and a quick 
rinse with distilled water, grids were stained with a solution consisting of 9 parts 2\% Methyl Cellulose and 1 part 3\% Uranyl acetate (both aqueous solutions).

Isolation of human B cell. To isolate human memory and naïve B cells from 14 healthy individuals and $18 \mathrm{HCV}$ patients, EasySep Human Memory B Cell Isolation Kit and EasySep Human Naïve B Cell Enrichment Kit (STEMCELL Technologies, Co. Ltd, USA) were used, respectively according to instruction manuals. IRB approval is obtained by University committee. Exosome isolation is described in Supplementary Methods.

Immunoblot. Proteins were detected by designated antibodies as following: MDA5 (D74E4, Rabbit mAb, Cell Signaling \#5321S, 1:1,000), MDA5 (Santa Cruz, sc-48031, 1:1,000), RIG-I rabbit Ab (Cell Signaling, \#4520S, 1:1,000), RIG-I (Santa Cruz, sc-48929, 1:1,000), B7-2 (BU63, sc-19617, Santa Cruz Biotechnology, 1:1,000), B7.2 (Santa Cruz, sc-28347, 1:1,000), Claudin-1 mAb (ZYMED, \#37-4900, 1:2,000). HCV E2 was detected by CBH5 or CBH17 antibodies (kind gifts from Prof. Steven Foung in Stanford University, Palo Alto, CA, USA, 1:1,000). The phosphorylation of PLC $\gamma 2$ (Tyr ${ }^{1217}$ : Cell Signaling cat\#: $\left.3871 \mathrm{~T}, 1: 2,000\right)$ and PKC $\alpha / \beta$ II (Thr ${ }^{638 / 641}$ : Cell Signaling cat\#: 9375 T, 1:2,000) were analysed using antibodies directed against their respective phosphorylated forms. Total protein levels were determined using antibodies not specific for the phosphorylated forms of these proteins. Uncropped scans of western blots are provided in Supplementary Fig. 10.

Co-receptor cloning by lentivirus B-cell cDNA library screen. To identify the possible novel receptor specific for the infection of lymphotropic HCV, we performed a lentivirus-based screen of a cDNA library, derived from the highly HCV-permissive B lymphoma Raji cell lines, for genes that render the non-permissive CD81 ${ }^{+}$SR-BI ${ }^{+}$HEK293T cell line infectable with HIV-1 particles pseudo-typed with HCVgp (HCVpp). More details are described in Supplementary Methods.

RNA synthesis. XbaI-digested constructs were treated with mung bean nuclease (New England Biolabs) to remove 4 nucleotides and leave the correct $3^{\prime}$ end of the HCV cDNA. Digested plasmid DNAs were purified and used as templates for RNA synthesis. The HCV RNA was synthesized in vitro using the MEGAscript T7 kit (Ambion, Austin, TX, USA). Synthesized RNA was treated with DNaseI (RQ1 RNase-free DNase, Promega) followed by acid phenol extraction to remove any remaining template DNA

Baculovirus expression and purification of HCV E2 proteins. E2 sequences from a genotype $2 \mathrm{~b}$ isolate (strain $\mathrm{SB}$ ) and genotype $2 \mathrm{a}$ isolate (strain JFH1) without the C-terminal transmembrane domains but containing a His6 tag at the $\mathrm{C}$ termini were cloned into a transfer vector (pBlueBacHis2; Invitrogen, Carlsbad, CA, USA). Expression of recombinant E2 proteins in insect cells (Sf9) was performed as described in the Bac-N-Blue Baculovirus Expression System (Invitrogen) and purified as previously described ${ }^{79}$. Briefly, insect Sf 9 cells were grown in Grace's Insect medium supplemented with $10 \% \mathrm{FBS}$ at $28^{\circ} \mathrm{C}$. The bacmid DNAs were transfected into Sf 9 cells; the virus generated was amplified two more times at a low multiplicity of infection before being used for infection of Sf 9 cells (at high multiplicity of infection). At $72 \mathrm{~h}$ post-infection, cells were lysed in $50 \mathrm{mM}$ Tris- $\mathrm{HCl}(\mathrm{pH} 8.5), 0.15 \mathrm{M} \mathrm{NaCl}, 0.1 \%$ Triton X-100 and protease inhibitor cocktail (Roche, Basel, Switzerland) and then sonicated and centrifuged at 100,000 g. E2 were purified from the crude lysates by using HiTrap chelating columns (Amersham Biosciences, Piscataway, NJ, USA) loaded with $0.1 \mathrm{M} \mathrm{NiCl}$ (ref. 80). The extracts were diluted in $50 \mathrm{mM}$ sodium phosphate (pH 8), $300 \mathrm{mM} \mathrm{NaCl}$ and $10 \%$ glycerol (buffer A) and bound to the column (flow rate: $0.5 \mathrm{ml} \mathrm{min}^{-1}$ ). The column was washed sequentially with buffer A, buffer B $(50 \mathrm{mM}$ sodium phosphate [pH 6], $300 \mathrm{mM} \mathrm{NaCl}, 10 \%$ glycerol) and buffer $\mathrm{C}$ ( $50 \mathrm{mM}$ sodium phosphate [pH 8], $1 \mathrm{M} \mathrm{NaCl}, 10 \%$ glycerol). The proteins were eluted in buffer A containing $200 \mathrm{mM}$ imidazole and buffer exchanged into phosphate-buffered saline without $\mathrm{Ca} 2+$ and $\mathrm{Mg} 2+[\mathrm{PBS}(-)]$ plus $10 \%$ glycerol.

Statistical analysis. ANOVA analysis was used for multiple comparisons. For correlation analysis, Pearson's correlation test was performed in GraphPad Prism software. For all other statistical analyses, the nonparametric Mann-Whitney test or two-tailed $t$-test was employed. Values of $P<0.05$ were considered to be statistically significant.

Data availability. The sequence of the HCV SB clone has been deposited in the GenBank Nucleotide database with the accession code KM349851. The authors declare that all other relevant data are available from the authors on request.

\section{References}

1. Agnello, V., Chung, R. T. \& Kaplan, L. M. A role for hepatitis C virus infection in type II cryoglobulinemia. N. Engl. j. med. 327, 1490-1495 (1992).
2. Idilman, R. et al. Lymphoproliferative disorders in chronic hepatitis C. J. Viral Hepat. 11, 302-309 (2004)

3. Zuckerman, E. et al. Hepatitis C virus infection in patients with B-cell non-Hodgkin lymphoma. Ann. Intern. Med. 127, 423-428 (1997).

4. Silvestri, F. et al. Prevalence of hepatitis C virus infection in patients with lymphoproliferative disorders. Blood 87, 4296-4301 (1996).

5. Mele, A. et al. Hepatitis C virus and B-cell non-Hodgkin lymphomas: an Italian multicenter case-control study. Blood 102, 996-999 (2003).

6. Marukian, S. et al. Cell culture-produced hepatitis C virus does not infect peripheral blood mononuclear cells. Hepatology 48, 1843-1850 (2008).

7. Stamataki, Z. et al. Hepatitis C virus association with peripheral blood B lymphocytes potentiates viral infection of liver-derived hepatoma cells. Blood 113, 585-593 (2009).

8. Kondo, Y. et al. Hepatitis $\mathrm{C}$ virus infection of $\mathrm{T}$ cells inhibits proliferation and enhances fas-mediated apoptosis by down-regulating the expression of CD44 splicing variant 6. J. infect. dis. 199, 726-736 (2009).

9. Kondo, Y. et al. HCV infection enhances Th17 commitment, which could affect the pathogenesis of autoimmune diseases. PLoS ONE 9, e98521 (2014).

10. Wunschmann, S., Medh, J. D., Klinzmann, D., Schmidt, W. N. \& Stapleton, J. T. Characterization of hepatitis $\mathrm{C}$ virus (HCV) and HCV E2 interactions with CD81 and the low-density lipoprotein receptor. J. virol. 74, 10055-10062 (2000).

11. Scarselli, E. et al. The human scavenger receptor class B type I is a novel candidate receptor for the hepatitis C virus. $E M B O$ j. 21, 5017-5025 (2002).

12. Pileri, P. et al. Binding of hepatitis C virus to CD81. Science 282, 938-941 (1998).

13. Ploss, A. et al. Human occludin is a hepatitis $C$ virus entry factor required for infection of mouse cells. Nature 457, 882-886 (2009).

14. Lupberger, J. et al. EGFR and EphA2 are host factors for hepatitis C virus entry and possible targets for antiviral therapy. Nat. med. 17, 589-595 (2011).

15. Martin, D. N. \& Uprichard, S. L. Identification of transferrin receptor 1 as a hepatitis C virus entry factor. Proc Natl Acad. Sci. USA 110, 10777-10782 (2013).

16. Evans, M. J. et al. Claudin-1 is a hepatitis $C$ virus co-receptor required for a late step in entry. Nature 446, 801-805 (2007).

17. Cocquerel, L., Voisset, C. \& Dubuisson, J. Hepatitis C virus entry: potential receptors and their biological functions. J. Gen. Virol. 87, 1075-1084 (2006).

18. Bartosch, B. et al. Cell entry of hepatitis $C$ virus requires a set of co-receptors that include the CD81 tetraspanin and the SR-B1 scavenger receptor. J. biol. chem. 278, 41624-41630 (2003).

19. Basu, A. et al. Sulfated homologues of heparin inhibit hepatitis C virus entry into mammalian cells. J. virol. 81, 3933-3941 (2007).

20. Dubuisson, J. Folding, assembly and subcellular localization of hepatitis $C$ virus glycoproteins. Curr. Top. Microbiol. Immunol. 242, 135-148 (2000).

21. Shimizu, Y. K., Iwamoto, A., Hijikata, M., Purcell, R. H. \& Yoshikura, H. Evidence for in vitro replication of hepatitis $\mathrm{C}$ virus genome in a human T-cell line. Proc. Natl Acad. Sci. USA 89, 5477-5481 (1992).

22. Ducoulombier, D. et al. Frequent compartmentalization of hepatitis $C$ virus variants in circulating B cells and monocytes. Hepatology 39, 817-825 (2004)

23. Di Liberto, G. et al. Clinical and therapeutic implications of hepatitis $C$ virus compartmentalization. Gastroenterology 131, 76-84 (2006).

24. Lerat, H., Shimizu, Y. K. \& Lemon, S. M. Cell type-specific enhancement of hepatitis $\mathrm{C}$ virus internal ribosome entry site-directed translation due to $5^{\prime}$ nontranslated region substitutions selected during passage of virus in lymphoblastoid cells. J. virol. 74, 7024-7031 (2000).

25. Nakajima, N., Hijikata, M., Yoshikura, H. \& Shimizu, Y. K. Characterization of long-term cultures of hepatitis C virus. J. virol. 70, 3325-3329 (1996).

26. Durand, T. et al. Occult infection of peripheral B cells by hepatitis C variants which have low translational efficiency in cultured hepatocytes. Gut 59, 934-942 (2010).

27. Laporte, J. et al. Comparative analysis of translation efficiencies of hepatitis $C$ virus $5^{\prime}$ untranslated regions among intraindividual quasispecies present in chronic infection: opposite behaviors depending on cell type. J. virol. 74, 10827-10833 (2000).

28. Friebe, P., Boudet, J., Simorre, J. P. \& Bartenschlager, R. Kissing-loop interaction in the $3^{\prime}$ end of the hepatitis $\mathrm{C}$ virus genome essential for RNA replication. J. virol. 79, 380-392 (2005).

29. Lagos-Quintana, M. et al. Identification of tissue-specific microRNAs from mouse. Curr. Biol. 12, 735-739 (2002).

30. Manfe, V. et al. miR-122 regulates p53/Akt signalling and the chemotherapyinduced apoptosis in cutaneous T-cell lymphoma. PLoS ONE 7, e29541 (2012)

31. Jopling, C. L., Yi, M., Lancaster, A. M., Lemon, S. M. \& Sarnow, P. Modulation of hepatitis C virus RNA abundance by a liver-specific MicroRNA. Science 309, 1577-1581 (2005)

32. Pedersen, I. M. et al. Interferon modulation of cellular microRNAs as an antiviral mechanism. Nature 449, 919-922 (2007).

33. Randall, G. et al. Cellular cofactors affecting hepatitis $\mathrm{C}$ virus infection and replication. Proc. Natl Acad. Sci. USA 104, 12884-12889 (2007). 
34. Valadi, H. et al. Exosome-mediated transfer of mRNAs and microRNAs is a novel mechanism of genetic exchange between cells. Nat. Cell Biol. 9, 654-659 (2007).

35. Lasser, C. et al. Human saliva, plasma and breast milk exosomes contain RNA: uptake by macrophages. J. Transl. Med. 9, 9 (2011).

36. Bala, S. et al. Circulating microRNAs in exosomes indicate hepatocyte injury and inflammation in alcoholic, drug-induced, and inflammatory liver diseases. Hepatology 56, 1946-1957 (2012).

37. Trebicka, J. et al. Hepatic and serum levels of miR-122 after chronic HCV-induced fibrosis. J. hepatol. 58, 234-239 (2012).

38. Starkey Lewis, P. J. et al. Circulating microRNAs as potential markers of human drug-induced liver injury. Hepatology 54, 1767-1776 (2011).

39. Wang, K. et al. Circulating microRNAs, potential biomarkers for drug-induced liver injury. Proc. Natl Acad. Sci. USA 106, 4402-4407 (2009).

40. Sung, V. M. et al. Establishment of B-cell lymphoma cell lines persistently infected with hepatitis $\mathrm{C}$ virus in vivo and in vitro: the apoptotic effects of virus infection. J. virol. 77, 2134-2146 (2003).

41. Wakita, T. et al. Production of infectious hepatitis $\mathrm{C}$ virus in tissue culture from a cloned viral genome. Nat. med. 11, 791-796 (2005).

42. Meylan, E. et al. Cardif is an adaptor protein in the RIG-I antiviral pathway and is targeted by hepatitis C virus. Nature 437, 1167-1172 (2005).

43. Li, X. D., Sun, L., Seth, R. B., Pineda, G. \& Chen, Z. J. Hepatitis C virus protease NS3/4A cleaves mitochondrial antiviral signaling protein off the mitochondria to evade innate immunity. Proc. Natl Acad. Sci. USA 102, 17717-17722 (2005).

44. Parvatiyar, K. \& Cheng, G. NOD so fast: NLRX1 puts the brake on inflammation. Immunity 34, 821-822 (2011).

45. Allen, I. C. et al. NLRX1 protein attenuates inflammatory responses to infection by interfering with the RIG-I-MAVS and TRAF6-NF-kappaB signaling pathways. Immunity 34, 854-865 (2011).

46. Saito, T. et al. Regulation of innate antiviral defenses through a shared repressor domain in RIG-I and LGP2. Proc. Natl Acad. Sci. USA 104, 582-587 (2007).

47. Sumpter, Jr R. et al. Regulating intracellular antiviral defense and permissiveness to hepatitis $\mathrm{C}$ virus RNA replication through a cellular RNA helicase, RIG-I. J. virol. 79, 2689-2699 (2005).

48. Pietschmann, T. et al. Construction and characterization of infectious intragenotypic and intergenotypic hepatitis $\mathrm{C}$ virus chimeras. Proc. Natl Acad. Sci. USA 103, 7408-7413 (2006).

49. Negre, D. et al. Lentiviral vectors derived from simian immunodeficiency virus. Curr. Top. Microbiol. Immunol. 261, 53-74 (2002).

50. Douam, F. et al. Specialization of Hepatitis C virus envelope glycoproteins for B lymphocytes in chronically infected patients. J. virol. 90, 992-1008 (2016).

51. Bartosch, B., Dubuisson, J. \& Cosset, F. L. Infectious hepatitis C virus pseudoparticles containing functional E1-E2 envelope protein complexes. J. exp. med. 197, 633-642 (2003).

52. Flint, M. et al. Characterization of hepatitis C virus E2 glycoprotein interaction with a putative cellular receptor, CD81. J. virol. 73, 6235-6244 (1999).

53. Yagnik, A. T. et al. A model for the hepatitis C virus envelope glycoprotein E2. Proteins 40, 355-366 (2000).

54. Owsianka, A., Clayton, R. F., Loomis-Price, L. D., McKeating, J. A. \& Patel, A. H. Functional analysis of hepatitis C virus E2 glycoproteins and virus-like particles reveals structural dissimilarities between different forms of E2. J. Gen. Virol. 82, 1877-1883 (2001).

55. Khan, A. G. et al. Structure of the core ectodomain of the hepatitis C virus envelope glycoprotein 2. Nature 509, 381-384 (2014).

56. Kohaar, I. et al. Splicing diversity of the human OCLN gene and its biological significance for hepatitis C virus entry. J. virol. 84, 6987-6994 (2010).

57. Lindenbach, B. D. et al. Complete replication of hepatitis $C$ virus in cell culture. Science 309, 623-626 (2005).

58. Liu, Y. J. et al. Memory B cells from human tonsils colonize mucosal epithelium and directly present antigen to T cells by rapid up-regulation of B7-1 and B7-2. Immunity 2, 239-248 (1995).

59. Lu, P. et al. CD40-mediated stimulation contributes to lymphocyte proliferation, antibody production, eosinophilia, and mastocytosis during an in vivo type 2 response, but is not required for T cell IL-4 production. J. immunol. 156, 3327-3333 (1996).

60. Sarhan, M. A., Chen, A. Y. \& Michalak, T. I. Differential expression of candidate virus receptors in human $\mathrm{T}$ lymphocytes prone or resistant to infection with patient-derived hepatitis C virus. PLoS ONE 8, e62159 (2013).

61. Gastaminza, P., Kapadia, S. B. \& Chisari, F. V. Differential biophysical properties of infectious intracellular and secreted hepatitis $\mathrm{C}$ virus particles. J. virol. 80, 11074-11081 (2006).

62. Reesink, H. W. et al. Rapid decline of viral RNA in hepatitis C patients treated with VX-950: a phase Ib, placebo-controlled, randomized study. Gastroenterology 131, 997-1002 (2006).
63. Charles, E. D. et al. Clonal B cells in patients with hepatitis $\mathrm{C}$ virus-associated mixed cryoglobulinemia contain an expanded anergic CD21low B-cell subset. Blood 117, 5425-5437 (2011).

64. Moir, S. et al. Evidence for HIV-associated B cell exhaustion in a dysfunctional memory B cell compartment in HIV-infected viremic individuals. J. exp. med. 205, 1797-1805 (2008).

65. Szocinski, J. L. et al. Activation-induced cell death of aggressive histology lymphomas by CD40 stimulation: induction of bax. Blood 100, 217-223 (2002).

66. Podojil, J. R., Kin, N. W. \& Sanders, V. M. CD86 and beta2-adrenergic receptor signaling pathways, respectively, increase Oct-2 and OCA-B expression and binding to the $3^{\prime}$-IgH enhancer in B cells. J. Biol. Chem. 279, 23394-23404 (2004).

67. Sahoo, N. C., Rao, K. V. \& Natarajan, K. CD80 expression is induced on activated B cells following stimulation by CD86. Scand. J. Immunol. 55, 577-584 (2002).

68. Kin, N. W. \& Sanders, V. M. CD86 stimulation on a B cell activates the phosphatidylinositol 3-kinase/Akt and phospholipase $\mathrm{C}$ gamma 2/protein kinase C alpha beta signaling pathways. J. immunol. 176, 6727-6735 (2006).

69. Bhatia, S., Edidin, M., Almo, S. C. \& Nathenson, S. G. B7-1 and B7-2: similar costimulatory ligands with different biochemical, oligomeric and signaling properties. Immunol. Lett. 104, 70-75 (2006).

70. Kondo, Y., Sung, V. M., Machida, K., Liu, M. \& Lai, M. M. Hepatitis C virus infects $\mathrm{T}$ cells and affects interferon-gamma signaling in $\mathrm{T}$ cell lines. Virology 361, 161-173 (2007).

71. Croft, M. \& Dubey, C. Accessory molecule and costimulation requirements for CD4 T cell response. Crit. Rev. Immunol. 17, 89-118 (1997).

72. Rosa, D. et al. Activation of naive B lymphocytes via CD81, a pathogenetic mechanism for hepatitis $\mathrm{C}$ virus-associated B lymphocyte disorders. Proc. Natl Acad. Sci. USA 102, 18544-18549 (2005).

73. Kasama, Y. et al. Persistent expression of the full genome of hepatitis $C$ virus in $\mathrm{B}$ cells induces spontaneous development of B-cell lymphomas in vivo. Blood 116, 4926-4933 (2010).

74. Laporte, J. et al. Differential distribution and internal translation efficiency of hepatitis $\mathrm{C}$ virus quasispecies present in dendritic and liver cells. Blood 101, 52-57 (2003).

75. Sarhan, M. A., Pham, T. N., Chen, A. Y. \& Michalak, T. I. Hepatitis C virus infection of human T lymphocytes is mediated by CD5. J. virol. 86, 3723-3735 (2012).

76. Rocha-Perugini, V. et al. The CD81 partner EWI-2wint inhibits hepatitis C virus entry. PLOS ONE 3, e1866 (2008).

77. Hueging, K. et al. Apolipoprotein E codetermines tissue tropism of hepatitis C virus and is crucial for viral cell-to-cell transmission by contributing to a postenvelopment step of assembly. J. virol. 88, 1433-1446 (2014).

78. Ishido, S. et al. Inhibition of natural killer cell-mediated cytotoxicity by Kaposi's sarcoma-associated herpesvirus K5 protein. Immunity 13, 365-374 (2000).

79. Machida, K., Cheng, K. T., Pavio, N., Sung, V. M. \& Lai, M. M. Hepatitis C virus E2-CD81 interaction induces hypermutation of the immunoglobulin gene in B cells. J. virol. 79, 8079-8089 (2005).

80. Niwa, M., Sidrauski, C., Kaufman, R. J. \& Walter, P. A role for presenilin-1 in nuclear accumulation of Ire1 fragments and induction of the mammalian unfolded protein response. Cell 99, 691-702 (1999).

\section{Acknowledgements}

We thank Dr S.M.T. (USC) for critical reading, Mr Jian-Chang Liu (USC) for technical assistance, Dr Steven Foung (Stanford University) for providing anti-E2 neutralizing antibody, Dr Rice (Rockefeller University) for Huh7.5 cells, Dr Chisari (Scripps Research Institute) for Huh7.5.1 cells, Dr Bartenschlager (University of Heidelberg) for HCV Con1 replicons, Dr Aizaki and other Lai lab members for invaluable suggestions, Dr Melvin Trousdale for electron microscopic analysis, Dr Nathaniel R. Landau for a luciferase construct, Dr Weeraratna from the National Institutes of Health (NIH) for Claudin-1 expression vector, Dr Ernesto Barron and Mr Anthony Rodriguez for Electron microscopy analysis, Mr Emmanuel V. Dimacali from University of Southern California for FACS analysis and Mr Chad Nakagawa (USC) for critical reading.

This project was supported by NIH grants 1R01AA018857-01, pilot project funding (5P30DK048522-13), P50AA11999 (Animal Core, Morphology Core, and Pilot Project Program), U19AI083025 and R24AA012885 (Non-Parenchymal Liver Cell Core). This research is also supported by a Research Scholar Grant, RSG-12-177-01-MPC and pilot funding (IRG-58-007-48) from the American Cancer Society. Microscopy was performed by the Cell and Tissue Imaging Core of the USC Research Center for Liver Diseases (P30 DK048522). Animal imaging was performed by the USC Molecular Imaging Center supported by NIH/NVRR S10. Tissue pathological slide preparation was performed by Ms Moli Chen of the Norris Comprehensive Cancer Center Translational Pathology Core. 


\section{Author contributions}

K.M. and M.M.C.L. conceived of the study. C.-L.C., K.M., J.Y.H., T.W. and Y.K. obtained the data. C.-L.C., K.M., J.S. and M.M.C.L. provided data management; T.W., F.-L.C. and L.S. provided essential reagents and specimens. K.M. and M.M.C.L. conducted the data analysis and drafted the report. All authors interpreted the data and contributed to the final version of this report.

\section{Additional information}

Supplementary Information accompanies this paper at http://www.nature.com/ naturecommunications

Competing financial interests: The authors declare no competing financial interests.

Reprints and permission information is available online at http://npg.nature.com/ reprintsandpermissions/
How to cite this article: Chen, C.-L. et al. Hepatitis C virus has a genetically determined lymphotropism through co-receptor B7.2. Nat. Commun. 8, 13882 doi: $10.1038 /$ ncomms13882 (2017)

Publisher's note: Springer Nature remains neutral with regard to jurisdictional claims in published maps and institutional affiliations.

(C) This work is licensed under a Creative Commons Attribution 4.0 International License. The images or other third party material in this article are included in the article's Creative Commons license, unless indicated otherwise in the credit line; if the material is not included under the Creative Commons license, users will need to obtain permission from the license holder to reproduce the material. To view a copy of this license, visit http://creativecommons.org/licenses/by/4.0/

(C) The Author(s) 2017 